\title{
De novo assembly and characterization of central nervous system transcriptome reveals neurotransmitter signaling systems in the rice striped stem borer, Chilo suppressalis
}

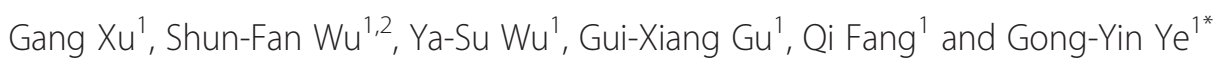

\begin{abstract}
Background: Neurotransmitter signaling systems play crucial roles in multiple physiological and behavioral processes in insects. Genome wide analyses of de novo transcriptome sequencing and gene specific expression profiling provide rich resources for studying neurotransmitter signaling pathways. The rice striped stem borer, Chilo suppressalis is a destructive rice pest in China and other Asian countries. The characterization of genes involved in neurotransmitter biosynthesis and transport could identify potential targets for disruption of the neurochemical communication and for crop protection.
\end{abstract}

Results: Here we report de novo sequencing of the C. suppressalis central nervous system transcriptome, identification and expression profiles of genes putatively involved in neurotransmitter biosynthesis, packaging, and recycling/degradation. A total of 54,411 unigenes were obtained from the transcriptome analysis. Among these unigenes, we have identified 32 unigenes (31 are full length genes), which encode 21 enzymes and 11 transporters putatively associated with biogenic aminergic signaling, acetylcholinergic signaling, glutamatergic signaling and GABAergic signaling. RT-PCR and qRT-PCR results indicated that 12 enzymes were highly expressed in the central nervous system and all the transporters were expressed at significantly high levels in the central nervous system. In addition, the transcript abundances of enzymes and transporters in the central nervous system were validated by qRT-PCR. The high expression levels of these genes suggest their important roles in the central nervous system.

Conclusions: Our study identified genes potentially involved in neurotransmitter biosynthesis and transport in C. suppressalis and these genes could serve as targets to interfere with neurotransmitter production. This study presents an opportunity for the development of specific and environmentally safe insecticides for pest control.

Keywords: Transcriptome, Central nervous system, Neurotransmitter signaling

\footnotetext{
* Correspondence: chu@zju.edu.cn

${ }^{1}$ State Key Laboratory of Rice Biology \& Key Laboratory of Agricultural

Entomology of Ministry of Agriculture, Institute of Insect Sciences, Zhejiang University, Hangzhou 310058, China

Full list of author information is available at the end of the article
}

( Biomed Central

(c) 2015 Xu et al. This is an Open Access article distributed under the terms of the Creative Commons Attribution License (http://creativecommons.org/licenses/by/4.0), which permits unrestricted use, distribution, and reproduction in any medium, provided the original work is properly credited. The Creative Commons Public Domain Dedication waiver (http:// creativecommons.org/publicdomain/zero/1.0/) applies to the data made available in this article, unless otherwise stated. 


\section{Background}

In both invertebrates and vertebrates, the control of physiology and behavior is achieved through the use of neurotransmitter signaling. While all tissues undoubtedly participate in this chemical communication, the central nervous system is a particularly rich source of neurotransmitter signaling compounds [1]. Neurotransmitters, as messengers in chemical synaptic transmission, play crucial roles in information transfer in the central nervous system [2]. In general, neurotransmitters consist of acetylcholine (ACh), glutamate (Glu), $\gamma$-aminobutyric acid (GABA), and monoamines such as dopamine (DA), octopamine (OA), tyramine (TA), serotonin (5-HT) and histamine (HA). Neurotransmitters are always produced via various synthetases in the presynaptic terminal to regulate multiple physiological and behavioral processes. Subsequently, neurotransmitters generated in the neurons are antiported by protons into synaptic vesicles using vesicular neurotransmitter transporters. Interactions of vesicles with the neurosynapse membrane occur when the calcium level inside the cell changes. These processes are assisted by vesicleassociated membrane proteins. The neurotransmitters are then released into synaptic cleft by fusion of the vesicles and cell membranes, which then interact with neurotransmitter receptors located in postsynaptic membranes [3]. In insects, the actions of neurotransmitters have been shown to be mainly mediated via the activation of ligand-gated ion channels and related G proteincoupled receptors (GPCRs) [4, 5]. A common feature of GPCR activation is the subsequent change of the intracellular concentration of second messengers, including cAMP and $\mathrm{Ca}^{2+}$. Some GPCRs bind to Gos/ Goi proteins, which then interact with adenylyl cyclase $(\mathrm{AC})$ in the plasma membrane to increase or decrease the concentration of cAMP. Some other GPCRs bind to Gaq subunits and activate phospholipase $\mathrm{C}$ (PLC) activity, causing a rise of $\left[\mathrm{Ca}^{2+}\right]_{\mathrm{i}}[6]$. In brief, the effects of the neurotransmitters are mediated through interactions with corresponding G protein-coupled receptors (GPCRs) to alter the concentrations of second messengers in the signaling pathways, resulting in modulation of various physiological processes. The actions of neurotransmitters are terminated by their reuptake to the cytosol via neurotransmitter transporters.

The rice striped stem borer, Chilo suppressalis (Walker) (Lepidoptera: Crambidae) is one of the most economically important rice pests in Asia, northern Africa, and southern Europe. It causes serious crop loss every year, particularly in China because of rice cultivation and the popularization of hybrid varieties. To date, chemical control is still the major method to protect rice from damage by the rice stem borer. Unfortunately, C. suppressalis has developed resistance to many chemical insecticides and the estimated cost for controlling this pest is approximately $160,000,000$ US dollars annually [7]. Compared to 10 years ago, the pesticides currently used in controlling the borers are much different. This is due to the evolution of multiple resistances to several classes of commonly used insecticides, including nereistoxin analogues, organochlorines, organophosphates, pyrethroids, and phenylpyrazoles [8]. The development of insecticide resistance in rice striped stem borer is the primary reason for insecticide replacement, along with the introduction of new chemical insecticides with higher activity [9]. Recently, some insecticides with novel mode of actions, such as chlorantraniliprole, have been widely applied in rice fields against rice borers [10]. Therefore, crop damage and high resistance emphasize the urgency for developing innovative control measures and resistance management strategies [7].

However, little is known about the neurotransmitter signaling systems in C. suppressalis. There were only some researches about octopamine, tyramine and acetylcholinesterases (AChEs) in C. suppressalis. Octopamine may play a role in mediating stress hormone effects on immune function via an $\alpha$-adrenergic-like octopamine receptor [11] and is involved in the regulation of locomotion through a $\beta$-adrenergic-like octopamine receptor [12]. The molecular and pharmacological characterization of two tyramine receptors and two splicing variants of $\alpha_{2}$-adrenergic like octopamine receptors with different signaling properties have been reported in $C$. suppressalis $[5,13,14]$. In addition, RNA interference of AChE1 and AChE2 reveals their different contributions to motor ability and larval growth in C. suppressalis [15]. In an attempt to provide a more complete foundation for future molecular and physiological investigation of neurotransmitter signaling in C. suppressalis, we have initiated the characterization of neurochemical signaling systems using de novo sequencing of central nervous system transcriptome. In the present study, we have identified genes encoding enzymes and transporters with putative functions in neurotransmitter signaling systems. Since these genes are associated with biosynthesis and transport of neurotransmitters, they are prime targets of pesticides, and our work provides a valuable molecular resource for developing new effective and specific drugs for insect pest control.

\section{Results and discussion}

\section{Illumina sequencing and unigenes assembly}

The transcriptomic sequence data were generated using a central nervous system cDNA library and Illumina HiSeq 2000 technology. For C. suppressalis, we acquired $142,051,094$ bp raw reads from central nervous system transcriptome. After eliminating adapters, ambiguous nucleotides and low quality sequences, 138,063,130 bp clean reads 
remained, which accumulated to a total of $13.8 \mathrm{~Gb}$ with a GC percentage of $43.91 \%$ (Table 1). Subsequently, C. suppressalis central nervous system transcriptome was de novo assembled using the short reads assembling programTrinity [16]. Total clean base pairs yield 105,769 transcripts with an N50 length of 2,647 bp and an N90 length of $512 \mathrm{bp}$. These transcripts range from 201 to over 21,491 bp with an average size of 1,330 bp (Additional file 1). Among the transcripts, 44,109 (41.7\%) are between 200 bp and 500 bp long, and $21.4 \%$ are over 2,000 bp (Fig. 1). After assembly of the transcripts into unigenes, 54,411 unigenes are obtained with an N50 length of 1,808 bp and an N90 length of $314 \mathrm{bp}$. These unigenes are from 201 to over 21,491 bp with an average size of $893 \mathrm{bp}$ (Additional file 1). Among the assembled unigenes, 31,510 (41.7\%) are between $200 \mathrm{bp}$ and $500 \mathrm{bp}$ long, and $11.6 \%$ are over 2,000 bp (Fig. 1). All sequences of the unigenes used in this study are provided in Additional file 2.

\section{Functional annotation by searching against public databases}

To validate and annotate the assembled unigenes, sequence similarity searches were conducted using the $\mathrm{Nr}$, Nt, KEGG, SwissProt, PFAM, GO, and COG databases [17-19] with an E-value threshold of $10^{-5}$. The results indicated that out of 54,411 unigenes, 19,148 (35.2\%), 3,945 (7.3 \%), 3,617 (6.6 \%), 11,863 (21.8\%), 14,454 (26.6\%), 15,725 (28.9\%), and 8,900 (16.4\%) unigenes showed significant similarity to known proteins in $\mathrm{Nr}$, Nt, KEGG, SwissProt, PFAM, GO, and COG databases, respectively. In addition, at 1,060 (1.9 \%) or 21,956 (40.4\%) unigenes were annotated in all or at least one database (Fig. 2). The E-value distribution of the top hits in the $\mathrm{Nr}$ database revealed that $56.02 \%$ of the mapped sequences showed significant homology matches $(<1.0 \mathrm{E}-50)$ (Fig. 3a). The similarity distribution showed that $7.83 \%$ of the sequences had $>95 \%$ homology, followed by $75.48 \%$ of the sequences with homology from $60 \%$ to $95 \%$. Only $16.68 \%$ of the

Table 1 The quality of C. suppressalis central nervous system unigene sequences and assembly

\begin{tabular}{ll}
\hline Raw reads (bp) & $142,051,094$ \\
\hline Clean reads (bp) & $138,063,130$ \\
Clean base pairs (Gb) & 13.8 \\
Error (\%) & 0.03 \\
Q20 (\%) & 98.00 \\
Q30 (\%) & 93.03 \\
GC (\%) & 43.91 \\
Unigenes & 54,411 \\
\hline
\end{tabular}

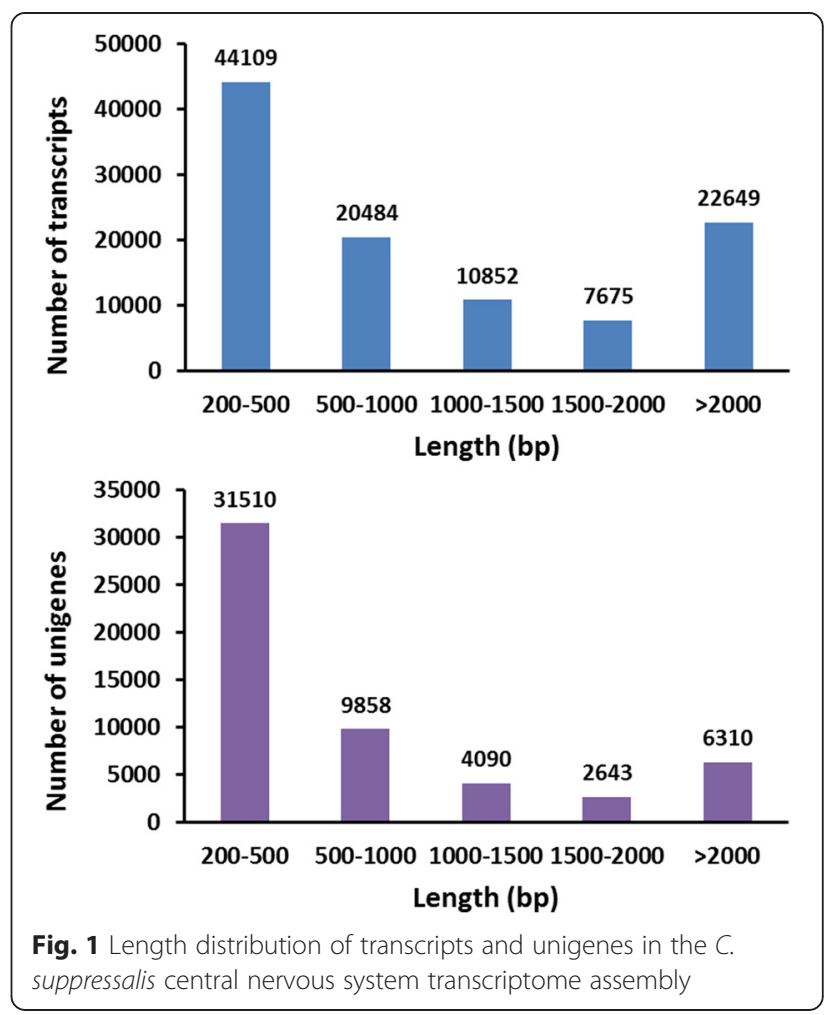

sequences had homology lower than $60 \%$ (Fig. 3b). Species specific distribution indicated that some of the $C$. suppressalis unigenes were homologous to those from more than one species, but most unigenes were homologous to Lepidoptera species with 12,013 hits among 19,148 BLASTn searches, including 9,339 (48.77\%) hits to Danaus plexippus, 1,394 (7.28 \%) to B. mori, followed by 908 to Tribolium castaneum, and 535 to Acyrthosiphon pisum. The top 10 insect species that have significant BLASTn hits are shown in Fig. 3c.

\section{Gene Ontology (GO) and clusters of orthologous groups (COG) classification in C. suppressalis central nervous system transcriptome}

Gene Ontology (GO) is an international standardized gene functional classification system which offers a dynamic-updated controlled vocabulary and a strictly defined concept to comprehensively describe the properties of genes and their products in any organism [20]. To further reveal their functions, GO assignments were used to classify $C$. suppressalis central nervous system unigenes. The 54,411 assembled unigenes were annotated into different functional groups according to Gene Ontology (GO) analysis. Some unigenes were annotated into more than one GO category. Of the 54,411 unigenes, 15,725 could be categorized into 54 functional 


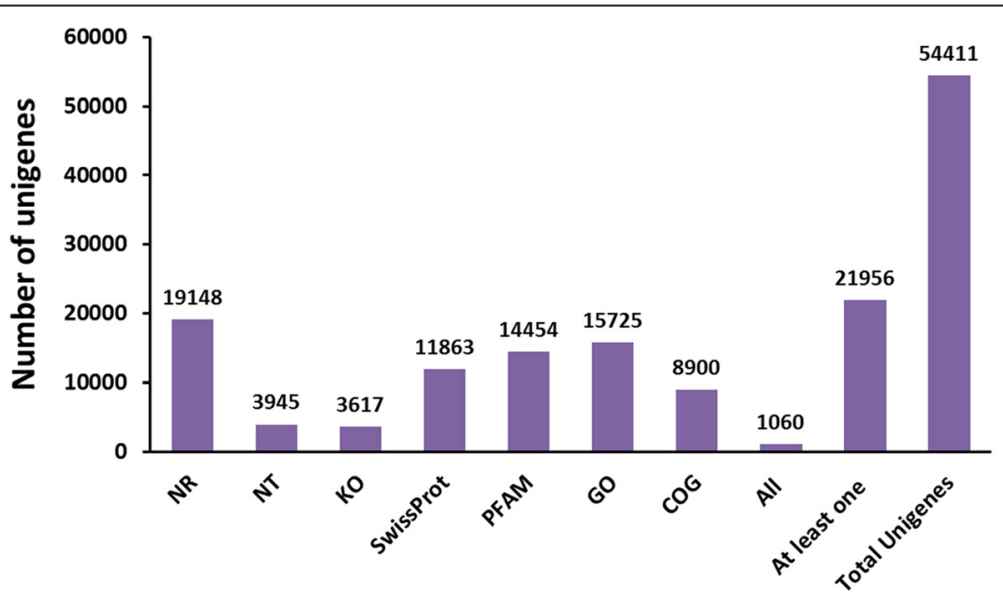

Fig. 2 The number of unigenes annotated in public database searched

groups (Additional file 3). The 'cellular process' and 'metabolic process' were most abundantly represented with 9,959 (66.33 \%) and 8,894 (55.56 \%) unigenes, respectively, within the 'biological process' GO ontology. In the 'cellular components' GO ontology the unigenes were mainly distributed in 'cell' (5,790 unigenes, $36.82 \%)$ and 'cell part' (5,789 unigenes, $36.81 \%)$. The GO analysis also showed that in the 'molecular function' ontology, 9,597 unigenes (61.03 \%) have 'binding' functions and 7,450 (47.38 \%) unigenes with 'catalytic activity' (Additional file 3).
The Clusters of Orthologous Groups (COG) is a database where the orthologous gene products are classified. Every protein in the COG database is assumed to be evolved from an ancestor protein, and the whole database is built on coding proteins with complete genome as well as systematic evolution relationships of bacteria, algae and eukaryotes [20]. All unigenes were aligned to the COG database to predict and classify potential functions. In total, 8,900 genes were assigned to the 25 COG classifications. Some unigenes were assigned to more than one COG category, thus a total
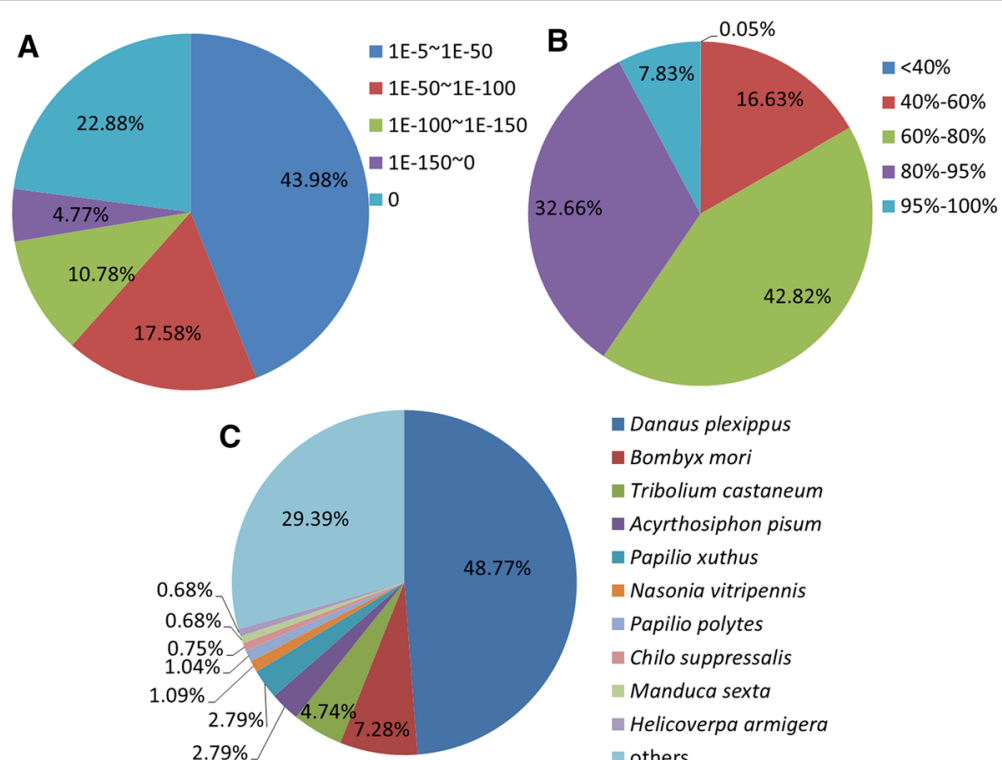

- Danaus plexippus

Bombyx mori

- Tribolium castaneum

- Acyrthosiphon pisum

Papilio xuthus

Nasonia vitripennis

Papilio polytes

- Chilo suppressalis

Manduca sexta

- Helicoverpa armigera

w others

Fig. 3 Characteristics of similarity search of unigenes against Nr database. (a) E-value distribution of BLAST hits for each unigene with a cut off E-value of 1.0E-5. (b) Similarity distribution of the top BLAST hits for each unigene. (c) Species distribution of the top BLAST hits for each unigene in Nr database 
of 9,920 sequences were assigned to 25 COG categories. Among the 25 COG categories, the cluster of 'General function prediction only' $(2,276,25.57 \%)$ was the largest group, followed by 'Signal transduction' $(1,170$, $13.15 \%)$, 'Post-translational modification, protein turnover, chaperon' (831, 9.34 \%), 'Transcription' (574, $6.45 \%$ ), and 'Function unknown' (514, $5.78 \%$ ), whereas only a few unigenes were assigned to 'Nuclear structure' and 'Cell motility' (Additional file 4).

\section{Metabolic pathway analysis by KEGG}

The Kyoto Encyclopedia of Genes and Genomes (KEGG) pathway database records the networks of molecular interactions in the cells, and variations of networks specific to particular organisms. Pathway-based analysis helps us to further understand the biological functions and interactions of genes [20]. In order to find out which biological pathways are active in C. suppressalis central nervous system, 54,411 unigenes were assigned to the reference pathways in KEGG. Consequently, 5,548 unigenes were mapped to 239 pathways. Among these pathways, 'Purine metabolism' (160 unigenes), 'Ribosome' (149 unigenes) and 'Protein processing in endoplasmic reticulum' (142 unigenes) (Fig. 4a) were the most common pathways in C. suppressalis central nervous system. Enrichment analysis is an effective way to identify the KEGG pathways that frequently occur in a tissue using the whole body transcriptome as background [21, 22]. In C. suppressalis, a total of 12 enriched KEGG pathways in central nervous system were identified (Additional file 5). Pathways like 'Signal transduction', 'Amino acid metabolism' and 'Nervous system' were enriched in C. suppressalis central nervous system. This is consistent with the principal function of insect central nervous system - uptake of neurotransmitters. In addition, 'Transport and catabolism' and 'Endocrine system' were also enriched. These transport-related pathways have been shown to be particularly crucial during the secretion of neurotransmitters and the formation of the action potential between the presynaptic terminal and the synaptic cleft. In the pathway 'Nervous system', 219 unigenes were assigned to 10 KEGG pathways, including 'Dopaminergic synapse' (60 unigenes), 'Glutamatergic synapse' (57), 'Neurotrophin signaling pathway' (53), 'Cholinergic synapse' (50), 'Synaptic vesicle cycle' (42), 'Long-term potentiation' (41), 'Retrograde endocannabinoid signaling' (38), 'GABAergic synapse' (37), 'Serotonergic synapse' (37), and 'Long-term depression' (27) (Fig. 4b).

\section{Candidate genes in C. suppressalis central nervous system with putative functions in neurotransmitter biosynthesis and transport}

The overall enzymatic steps during neurotransmitter signaling system in C. suppressalis are likely to be similar to those in the fruit fly Drosophila melanogaster and other insect species, which include neurotransmitter biosynthesis, packaging, and recycling/ degradation [1]. Therefore, we used deduced amino acid sequences of the genes associated with neurotransmitter biosynthesis and transport in D. melanogaster and other insects as queries for local BLAST to identify the candidate genes encoding enzymes and transporters in C. suppressalis. By homology search, we identified a number of unigenes in the $C$. suppressalis central nervous system putatively involved in biogenic aminergic signaling, acetylcholinergic signaling, glutamatergic signaling and GABAergic signaling, including unigenes putatively encoding 21 enzymes (Table 2) and 11 transporters (Table 3). In addition, we further confirmed the enzymes and transporters in C. suppressalis by comparing them with other known
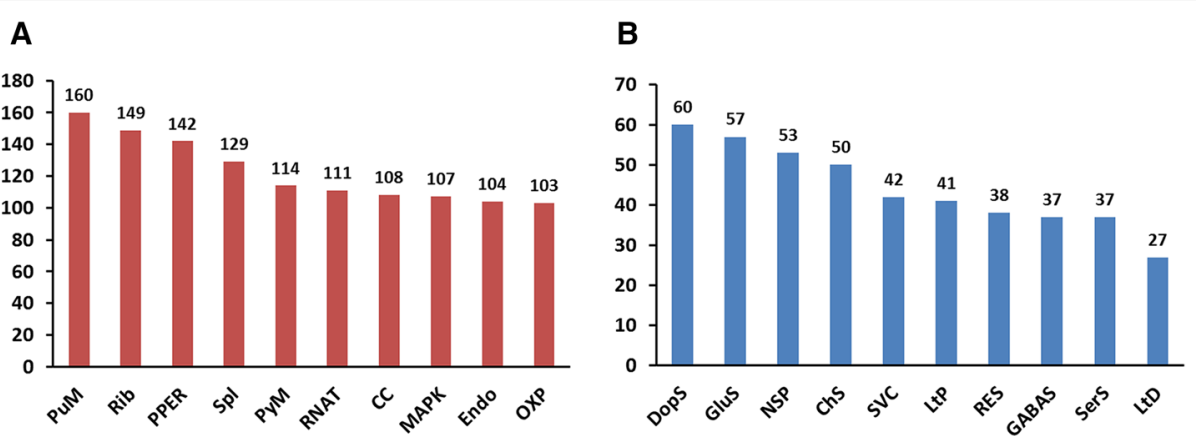

Fig. 4 Distribution of unigenes among the KEGG pathways. (a) The top ten KEGG pathways with highest numbers of unigenes in C. suppressalis central nervous system. (b) The KEGG pathways in the pathway 'Nervous system'. Abbreviation for pathways: Purine metabolism (PuM), Ribosome (Rib), Protein processing in endoplasmic reticulum (PPER), Spliceosome (Spl), Pyrimidine metabolism (PyM), RNA transport (RNAT), Cell cycle (CC), MAPK signaling pathway (MAPK), Endocytosis (Endo), Oxidative phosphorylation (OXP); Dopaminergic synapse (DopS), Glutamatergic synapse (GluS), Neurotrophin signaling pathway (NSP), Cholinergic synapse (ChS), Synaptic vesicle cycle (SVC), Long-term potentiation(LtP), Retrograde endocannabinoid signaling (RES), GABAergic synapse (GABAS), Serotonergic synapse (SerS), Long-term depression (LtD) 
Table 2 The enzymes involved in the biosynthesis pathway of neurotransmitters identified from C. suppressalis central nervous system transcriptome

\begin{tabular}{|c|c|c|c|c|c|c|c|c|c|c|}
\hline Unigene & Gene & Accession No. & Length & ORF & Putative identification & Species & Accession No. & Score & E-value & Identity \\
\hline comp54907_c0 & $\mathrm{TH}$ & KP657623 & 2232 & 1683 & tyrosine hydroxylase & Spodoptera exigua & AFG25778.1 & 1108 & 0 & $94 \%$ \\
\hline comp48842_c0 & DDC & KP657625 & 2590 & 1440 & dopa decarboxylase & Antheraea pernyi & AAR23825.1 & 906 & 0 & $89 \%$ \\
\hline comp56513_c0 & ebony & KP657628 & 3245 & 2586 & ebony & Papilio xuthus & BAE43845.2 & 1435 & 0 & $79 \%$ \\
\hline comp71328_c0 & $\tan$ & KP657627 & 1994 & 1218 & tan protein & Bombyx mori & NP_001170882.1 & 617 & 0 & $74 \%$ \\
\hline comp75401_c0 & aaNAT & KP657626 & 1856 & 783 & $\begin{array}{l}\text { arylalkylamine } \\
\mathrm{N} \text {-acetyltransferase }\end{array}$ & Bombyx mori & NP_001073122.1 & 373 & 0 & $72 \%$ \\
\hline comp54468_c0 & TDC & KP657629 & 2075 & 1878 & $\begin{array}{l}\text { aromatic amino acid } \\
\text { decarboxylase }\end{array}$ & Danaus plexippus & EHJ72689.1 & 1075 & 0 & $85 \%$ \\
\hline comp60420_c0 & $\mathrm{T} \beta \mathrm{H}$ & KP657630 & 3530 & 1755 & tyramine beta hydroxylase & Bombyx mori & NP_001243923.1 & 910 & 0 & $73 \%$ \\
\hline comp51351_c0 & $\mathrm{TRH}$ & KP657632 & 1875 & 1590 & tryptophan hydroxylase & Bombyx mori & XP_004929955.1 & 752 & 0 & $89 \%$ \\
\hline comp44225_c0 & $\mathrm{TPH}$ & KP657631 & 2087 & 1365 & phenylalanine hydroxylase & Papilio xuthus & BAE66652.1 & 851 & 0 & $88 \%$ \\
\hline comp52601_c0 & HDC & KP657633 & 2475 & 1773 & histidine decarboxylase & Danaus plexippus & ЕНJ77965.1 & 1043 & 0 & $85 \%$ \\
\hline comp36379_c0 & ChAT & KP657655 & 833 & & choline acetyltransferase & Bombyx mori & BAO23491.1 & 355 & $\begin{array}{l}4 \mathrm{E}- \\
114\end{array}$ & $83 \%$ \\
\hline comp63891_c2 & AChE1 & KP657634 & 5043 & 2085 & acetylcholinesterase 1 & Chilo suppressalis & ABO38111.1 & 1443 & 0 & $99 \%$ \\
\hline comp66146_c1 & AChE2 & KP657635 & 2255 & 1917 & acetylcholinesterase 2 & Chilo suppressalis & ABR24230.1 & 1334 & 0 & $100 \%$ \\
\hline comp57261_c0 & GLS & KP657636 & 3061 & 1932 & putative glutaminase & Danaus plexippus & EHJ71111.1 & 1170 & 0 & $87 \%$ \\
\hline comp56484_c0 & GS1 & KP657637 & 1379 & 1215 & glutamine synthetase 1 & Bombyx mori & XP_004930366.1 & 647 & 0 & $75 \%$ \\
\hline comp54696_c0 & GS2 & KP657638 & 5657 & 1143 & glutamine synthetase 2 & Papilio xuthus & BAM17922.1 & 706 & 0 & $93 \%$ \\
\hline comp52410_c0 & $\mathrm{GDH}$ & KP657639 & 2318 & 1665 & glutamate dehydrogenase & Papilio polytes & BAM20330.1 & 1115 & 0 & $95 \%$ \\
\hline comp63917_c0 & GAD1 & KP657640 & 3698 & 1623 & glutamate decarboxylase-like & Bombyx mori & XP_004925034.1 & 984 & 0 & $93 \%$ \\
\hline comp56481_c0 & GAD2 & KP657641 & 2518 & 1530 & black & Biston betularia & AEP43793.2 & 941 & 0 & $86 \%$ \\
\hline comp52445_c0 & GABAT & KP657642 & 3275 & 1482 & $\begin{array}{l}\text { 4-aminobutyrate } \\
\text { aminotransferase }\end{array}$ & Danaus plexippus & EHJ72994.1 & 771 & 0 & $74 \%$ \\
\hline comp58969_c0 & SSADH & KP657643 & 2049 & 1521 & $\begin{array}{l}\text { succinate-semialdehyde } \\
\text { dehydrogenase }\end{array}$ & Bombyx mori & XP_004932642.1 & 833 & 0 & $77 \%$ \\
\hline
\end{tabular}

insect enzymes and transporters involved in neurotransmitter biosynthesis and transport using phylogenetic tree analysis (Figs. 5, 6 and 7). Their relative transcript abundances in the central nervous system were detected by qRT-PCR (Fig. 8). We further validated and characterized the expression levels of these genes by RT-PCR and qRT-PCR in various tissues and the results were discussed below.

\section{Biogenic aminergic signaling}

Biogenic amines are one class of signaling molecules used by both vertebrate and invertebrate nervous systems, and they play a key role in regulating and modulating various physiological and behavioral processes. In insects, five biogenic amines are generally recognized: dopamine, octopamine, tyramine, serotonin and histamine [23].

\section{Dopaminergic signaling}

Dopamine is found at a relatively high level throughout the insect nervous system and is the most abundant monoamine present [24]. In insects, dopamine modulates various aspects of behavior such as locomotor activity [25], decision-making [26], phase change [27], copulation persistence [28], sucrose acceptance [29], learning and memory [30], and is also a precursor of melanin. To produce dopamine, tyrosine is first converted to L-3, 4-dihydroxyphenylalanine (L-DOPA) via the action of tyrosine hydroxylase $(\mathrm{TH})$, which is then converted to dopamine by DOPA decarboxylase (DDC) [31]. Dopamine is also utilized by NBAD synthase (ebony) and arylalkylamine $\mathrm{N}$-acetyl transferase (aaNAT) to produce pigments other than melanin. NBAD hydrolase $(\tan )$ catalyzes the reaction in the opposite direction to ebony, increasing dopamine concentration [32] (Fig. 9a). Dopamine is released as a neurotransmitter from synaptic vesicles via exocytosis at presynaptic terminal [33]. Subsequently, the reuptake of dopamine from the synaptic cleft can be accomplished through its interaction with plasma membrane monoamine transporter - dopamine transporter (DAT) [34]. Next, the refluent dopamine can be transported to synaptic vesicles 
Table 3 The transporters involved in the neurotransmitter signaling pathways identified from C. suppressalis central nervous system transcriptome

\begin{tabular}{|c|c|c|c|c|c|c|c|c|c|c|}
\hline Unigene & Gene & $\begin{array}{l}\text { Accession } \\
\text { No. }\end{array}$ & Length & ORF & Putative identification & Species & Accession No. & Score & E-value & Identity \\
\hline comp66192_c1 & DAT & KP657644 & 2448 & 1839 & dopamine transporter & Bombyx mori & NP_001037362.1 & 1076 & 0 & $90 \%$ \\
\hline comp62798_c0 & OAT & KP657645 & 2908 & 2250 & $\begin{array}{l}\text { high-affinity octopamine } \\
\text { transporter }\end{array}$ & Ostrinia nubilalis & AAZ08592.2 & 1340 & 0 & $91 \%$ \\
\hline comp64226_c0 & SERT & KP657646 & 3079 & 1782 & serotonin transporter & Bombyx mori & NP_001037436.1 & 1071 & 0 & $90 \%$ \\
\hline comp61773_c0 & VMAT & KP657647 & 1982 & 1458 & $\begin{array}{l}\text { synaptic vesicular amine } \\
\text { transporter }\end{array}$ & $\begin{array}{l}\text { Camponotus } \\
\text { floridanus }\end{array}$ & EFN70897.1 & 625 & 0 & $73 \%$ \\
\hline comp60635_c0 & ChT & KP657648 & 4324 & 1782 & high-affinity choline transporter & Trichoplusia ni & AAT88074.1 & 1091 & 0 & $93 \%$ \\
\hline comp63510_c0 & VAChT & KP657649 & 4635 & 1863 & $\begin{array}{l}\text { vesicular acetylcholine } \\
\text { transporter-like }\end{array}$ & Bombyx mori & NP_001275599.1 & 1070 & 0 & $87 \%$ \\
\hline comp71360_c0 & EAAT1 & KP657650 & 1655 & 1452 & $\begin{array}{l}\text { excitatory amino acid } \\
\text { transporter } 1\end{array}$ & Trichoplusia ni & AAB84380.1 & 839 & 0 & $86 \%$ \\
\hline comp63601_c1 & EAAT2 & KP657651 & 2956 & 1623 & $\begin{array}{l}\text { excitatory amino acid } \\
\text { transporter } 2\end{array}$ & Bombyx mori & NP_001240825.1 & 741 & 0 & $83 \%$ \\
\hline comp52964_c0 & VGluT & KP657652 & 2707 & 1782 & $\begin{array}{l}\text { vesicular glutamate } \\
\text { transporter }\end{array}$ & Bombyx mori & XP_004925576.1 & 972 & 0 & $85 \%$ \\
\hline comp65336_c0 & GAT & KP657653 & 5728 & 1824 & $\begin{array}{l}\text { high affinity GABA } \\
\text { transporter }\end{array}$ & Trichoplusia ni & AAF70819.1 & 1179 & 0 & $96 \%$ \\
\hline comp62581_c0 & VGAT & KP657654 & 2512 & 1617 & vesicular GABA transporter & Danaus plexippus & EHJ77951.1 & 947 & 0 & $88 \%$ \\
\hline
\end{tabular}

for storage through vesicular monoamine transporter(VMAT), which is also likely to function as a vesicular transporter for the storage of serotonin and octopamine [33, 35].

To elucidate the molecular basis of the dopaminergic signaling system, we identified dopamine-related genes involved in biosynthesis, signal transduction, and reuptake in C. suppressalis central nervous system transcriptome. We obtained five enzymes and two transporters, $\mathrm{TH}$, DDC, aaNAT, ebony, tan, DAT and VMAT. Interestingly, $\mathrm{TH}$ has two splicing variants, the long isoform has an open reading frame (ORF) of 1,683 bp and the deduced amino acid sequence shows $94 \%$ identity to $\mathrm{TH}$ of Spodoptera exigua (Protein ID: AFG25778.1), whereas the short isoform has an ORF of 1,527 bp. The alternative splicing mechanism has also been found in Mythimna separata and D. melanogaster [36]. Multiple sequence alignment showed that the alternative splicing domains were conserved in THs (Additional file 6). DDC contains an open reading frame of $1,440 \mathrm{bp}$ and its amino acid sequence is $89 \%$ identity to DDC of Antheraea pernyi (Protein ID: AAR23825.1). Comparison of $C$. suppressalis aaNAT with B. mori aaNAT (Protein ID: NP_001073122.1) revealed $72 \%$ identity in amino acid sequences. Two unigenes encoding ebony and tan proteins in C. suppressalis show $79 \%$ identity in amino acid sequence to ebony of Papilio xuthus (Protein ID: BAE43845.2 ) and $74 \%$ identity to tan protein of $B$. mori (Protein ID: NP_001170882.1), respectively (Table 2). In addition, two transporters, DAT and VMAT, show $90 \%$ and $73 \%$ identities in amino acid sequences with DAT of
B. mori (Protein ID: NP_001037362.1) and VMAT of Camponotus floridanus (Protein ID: EFN70897.1), respectively (Table 3 ). The RT-PCR and qRT-PCR results revealed that $\mathrm{TH}$ and $\mathrm{DDC}$ were highly expressed in the hemocytes in addition to central nervous system (Figs. 10a and 11). Exogenous stimuli could induce expression of $\mathrm{TH}$ and DDC, suggesting that dopamine may be an important molecule bridging the nervous system and immune system [37]. The expression level of aaNAT and ebony in the central nervous system was highest, while tan was expressed at the highest level in the gut (Figs. 10a and 11). Moreover, the RT-PCR and qRTPCR results showed that two transporters were expressed at significantly high levels in the central nervous system (Figs. 10c and 13), indicating that these two transporters are likely to play an vital role in regulating the storage and release of dopamine in the nervous system [35].

\section{Octopaminergic and tyraminergic signaling}

Octopamine is a biogenic amine with a widespread distribution in the insect central nervous system [38, 39]. The structures of octopamine and tyramine differ only in the respective presence or absence of a hydroxyl group at $\beta$-position in their side chains [40]. Octopamine and tyramine are considered to be the invertebrate counterparts of the vertebrate adrenergic transmitters, and the two phenolamines are the only biogenic amines whose physiological significance is presumably restricted to invertebrates $[5,13,41,42]$. They regulate various physiological functions in insects, such as addiction, circadian rhythms, endocrine secretion, aggression, egg- 


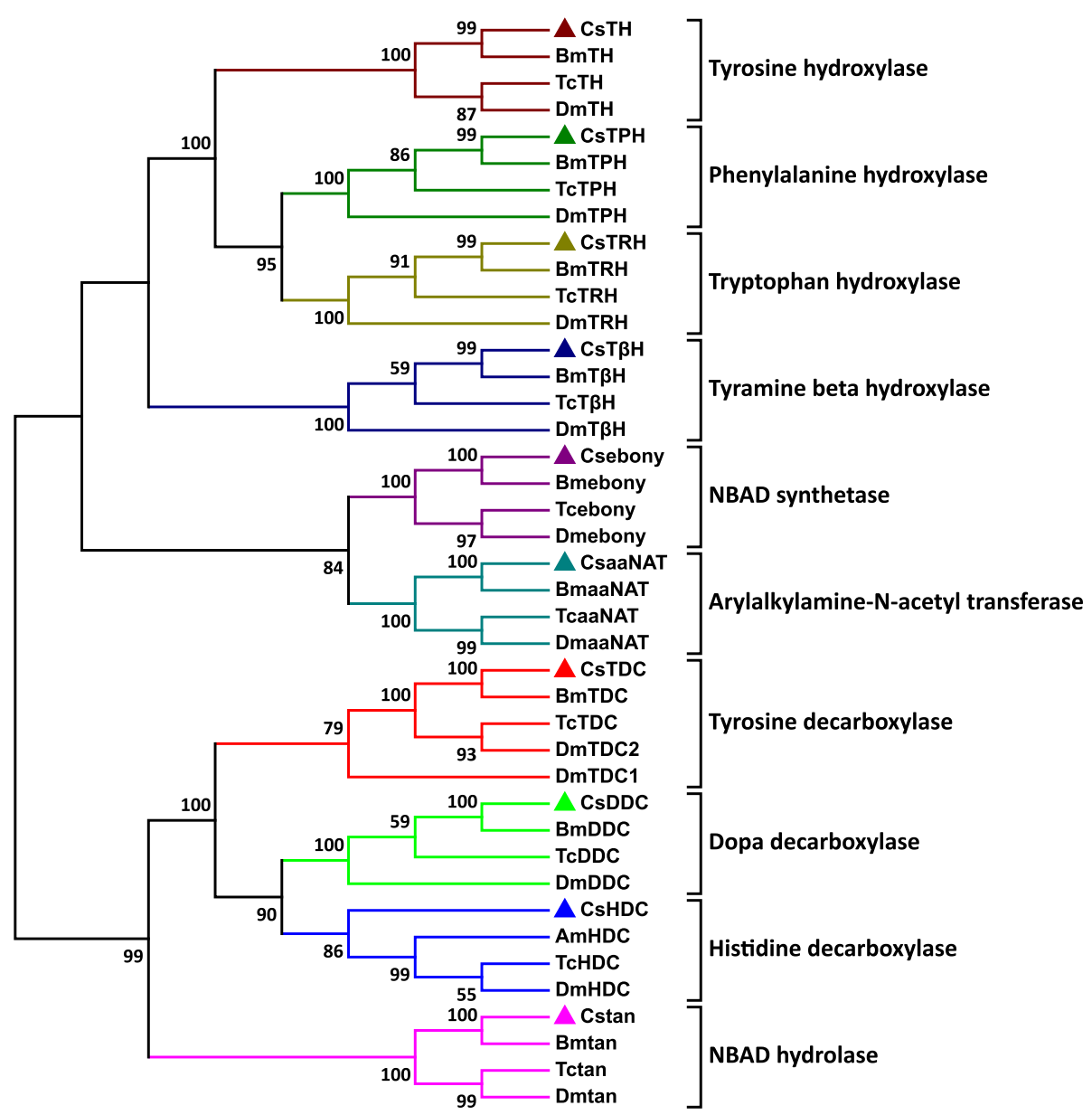

Fig. 5 The phylogenetic analysis of enzymes involved in the biosynthesis pathway of biogenic amines in insects. Included are C. suppressalis (Cs), B. mori (Bm), T. castaneum (Tc), D. melanogaster (Dm), and Apis mellifera (Am). The accession numbers of the squences are available in Additional file 11. Neighbor-joining trees were constructed using MEGA 5 software with 1000-fold bootstrap re-sampling. The numbers at the nodes of the branches represent the level of bootstrap support for each branch

laying, food-seeking, locomotion and decision-making $[12,43]$. For the production of octopamine, tyrosine is converted by tyrosine decarboxylase (TDC) to tyramine, which in turn is converted to octopamine via the action of tyramine beta hydroxylase $(\mathrm{T} \beta \mathrm{H})$ [31] (Fig. 9b). Octopamine and tyramine can be released from synaptic vesicles into synaptic cleft, then octopamine transporter acts in the reuptake system for both transmitters in the neurons involved in octopaminergic/tyraminergic signaling [24]. As mentioned above, vesicular monoamine transporter (VMAT) can function as a vesicular transporter for the storage of octopamine and tyramine [33].

To investigate the molecular basis of the octopaminergic/tyraminergic signaling system, we identified genes participating in octopamine/tyramine biosynthesis, signal transduction, and reuptake in C. suppressalis central nervous system transcriptome. We obtained two enzymes and one transporter, including TDC, T $\beta \mathrm{H}$ and OAT. TDC contains an open reading frame of 1,878 bp and the deduced amino acid sequence is $85 \%$ identity to TDC of $D$. plexippus (Protein ID: EHJ72689.1). The unigene with an open reading frame of 1,755 bp encodes a T $\beta \mathrm{H}$ with $73 \%$ identity to T $\beta \mathrm{H}$ of B. mori (Protein ID: NP_001243923.1) (Table 2). In addition, the transporter OAT shows $91 \%$ identity with OAT of Ostrinia nubilalis (Protein ID: AAZ08592.2) (Table 3). Unfortunately, no octopamine transporter has thus far been characterized from D. melanogaster. This is puzzling because both compounds must be removed following release. The only biogenic amine transporters found in Drosophila, DAT and SERT, have pharmacological features that are not tuned to take up these major neuroactive compounds. Although they are not for octopamine/tyramine uptake, these transporters are of great interest because they are prime targets for pharmacological compounds [41]. The RT-PCR and qRT- 


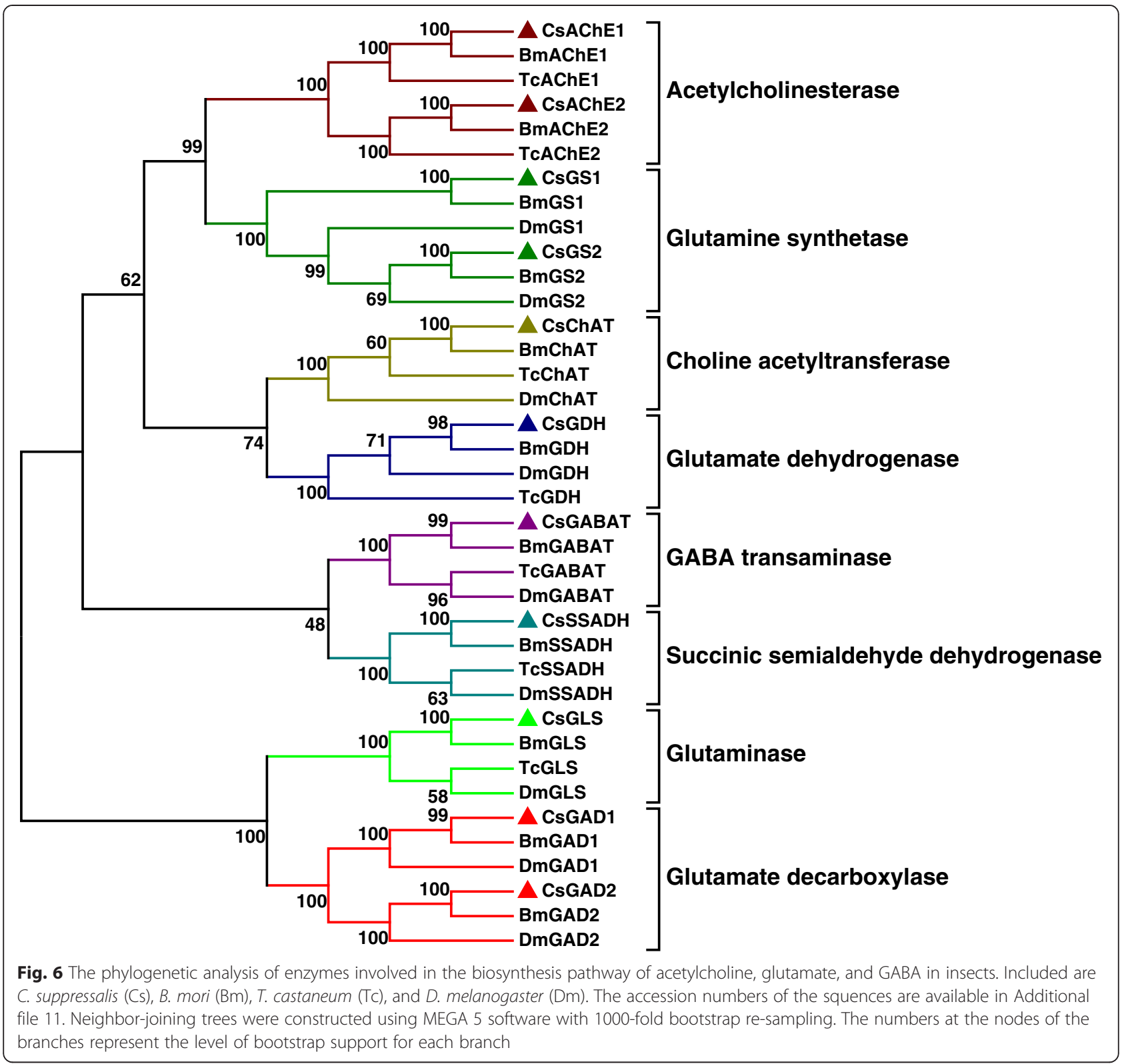

PCR results revealed that TDC, T $\beta \mathrm{H}$ and OAT were highly expressed in the central nervous system (Figs. 10a, $\mathrm{c}$ and 11,13$)$, suggesting that these genes are likely to play an important role in biosynthesis and transport of octopamine and tyramine in $C$. suppressalis central nervous system. Interestingly, octopamine also seems to play a role in the immune system of invertebrates, which resembles the innate immune system in humans, as its concentration in the hemolymph increases during an immune challenge [44]. In addition, a concentration-sensitive $\alpha$-adrenergiclike octopamine receptor is found on insect immune cells and plays a possible role in mediating stress hormone effects on immune function [11].

\section{Serotoninergic signaling}

The biogenic amine serotonin, or 5-hydroxytryptamine (5-HT), is widely distributed in animals. It acts through multiple receptors to modulate many complex behaviors in vertebrates and invertebrates [45]. In insects, serotonin signaling controls nutrition, heart rate, secretory processes, feeding, gut contraction, development, circadian rhythms, sleep, aggression, behavioral gregarization, phototactic behavior, learning, and memory [46, 47]. For the production of serotonin, tryptophan is converted to 5-hydroxytryptophan via either phenylalanine hydroxylase $(\mathrm{TPH})$ or tryptophan hydroxylase $(\mathrm{TRH})$, which in turn is converted to serotonin by DDC [31] (Fig. 9c). 


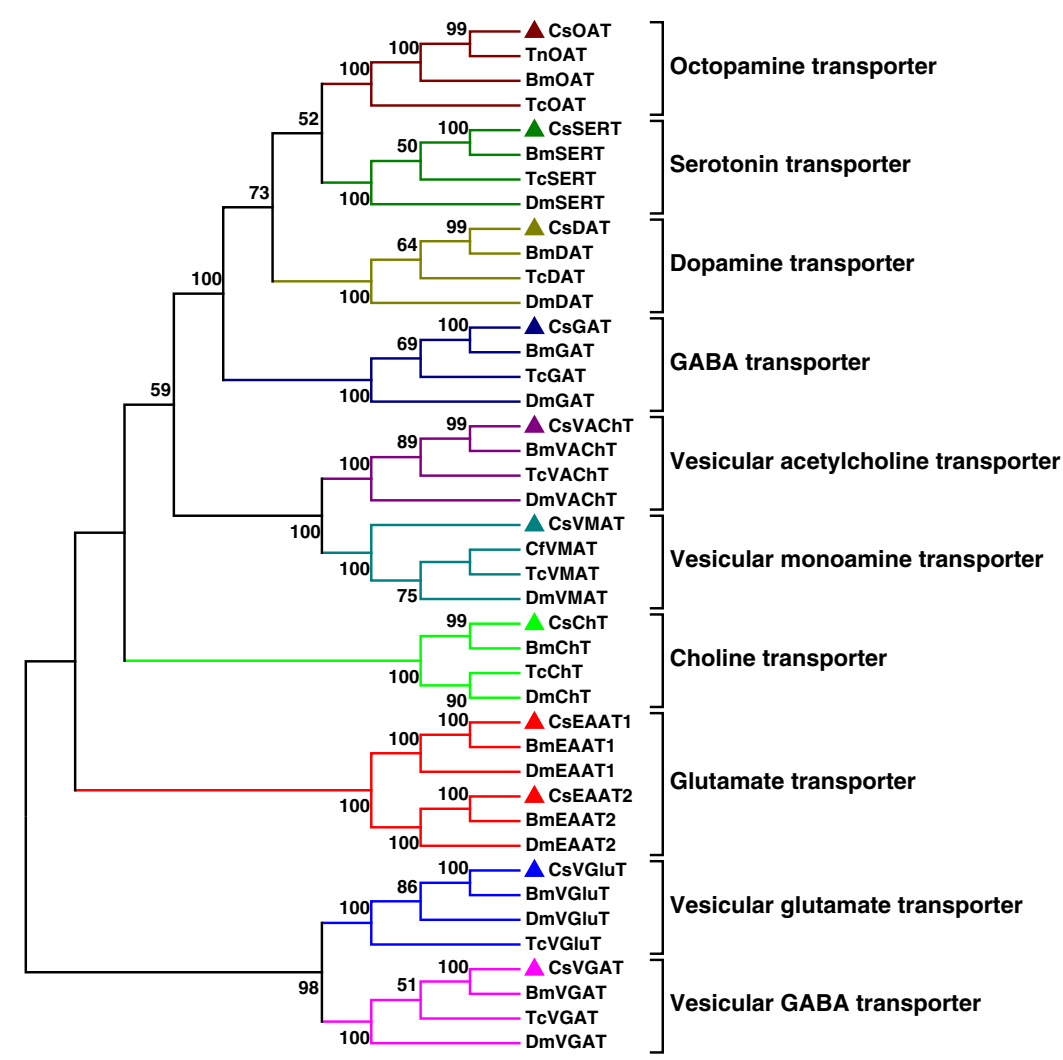

Fig. 7 The phylogenetic analysis of transporters involved in neurotransmitter signaling systems in insects. Included are C. suppressalis (Cs), T. ni (Tn), B. mori (Bm), T. castaneum (Tc), C. floridanus (Cf), and D. melanogaster (Dm). The accession numbers of the squences are available in Additional file 11. Neighbor-joining trees were constructed using MEGA 5 software with 1000-fold bootstrap re-sampling. The numbers at the nodes of the branches represent the level of bootstrap support for each branch

Serotonin is released from secretory vesicles into synaptic cleft like dopamine and octopamine, and serotonin transporter (SERT) can mediate reuptake as the primary extracellular mechanism for clearing of released serotonin, and SERT-mediated reuptake is critical for maintaining a high intracellular serotonin pool [48]. In $D$. melanogaster, tryptophan hydroxylase is encoded by two genes, dTPH and dTRH [49]. DDC is involved in the biosynthesis of not only serotonin but also dopamine, and thus DDC is expressed in serotoninergic and dopaminergic neurons in the central nervous system [50].

To illustrate the molecular basis of the serotoninergic signaling system, we identified serotonin-related genes associated with serotonin biosynthesis, signal transduction and reuptake in C. suppressalis central nervous system transcriptome. We obtained two enzymes and one transporter, including TPH, TRH and SERT. TPH contains an open reading frame of 1,365 bp with $88 \%$ identity in amino acid sequence to TPH of $P$. xuthus (Protein ID: BAE66652.1). The unigene with an open reading frame of 1,590 bp encodes a TRH with $89 \%$ identity to TRH of B. mori (Protein ID: XP_004929955.1) (Table 2). Moreover, the transporter SERT shows $90 \%$ identity in amino acid sequence with SERT of $B$. mori (Protein ID: NP_001037436.1) (Table 3). The RT-PCR and qRT-PCR results showed that TPH and TRH were highly expressed in the fat body (Figs. 10a and 11), while the transporter SERT was specifically expressed in the central nervous system (Figs. 10c and 13). It is interesting that serotonin synthesis and storage are also found in immune cells, and serotonin participates in innate immune response and adaptive immunity. Thus, serotonin also plays an important role in immune signaling outside of the central nervous system [51].

\section{Histaminergic signaling}

The biogenic amine histamine (HA) is among the first compounds recognized as a messenger molecule in intercellular communication [52]. In vertebrates, it functions as a transmitter in the brain as well as a transmitter, hormone and mediator in peripheral systems. In the periphery, histamine is responsible for different actions such as the contraction of smooth muscle, capillary dilation or stimulation of gastric secretion [53]. In invertebrates, histamine has various roles in neurotransmission in the brain, such as olfaction in crustaceans and 

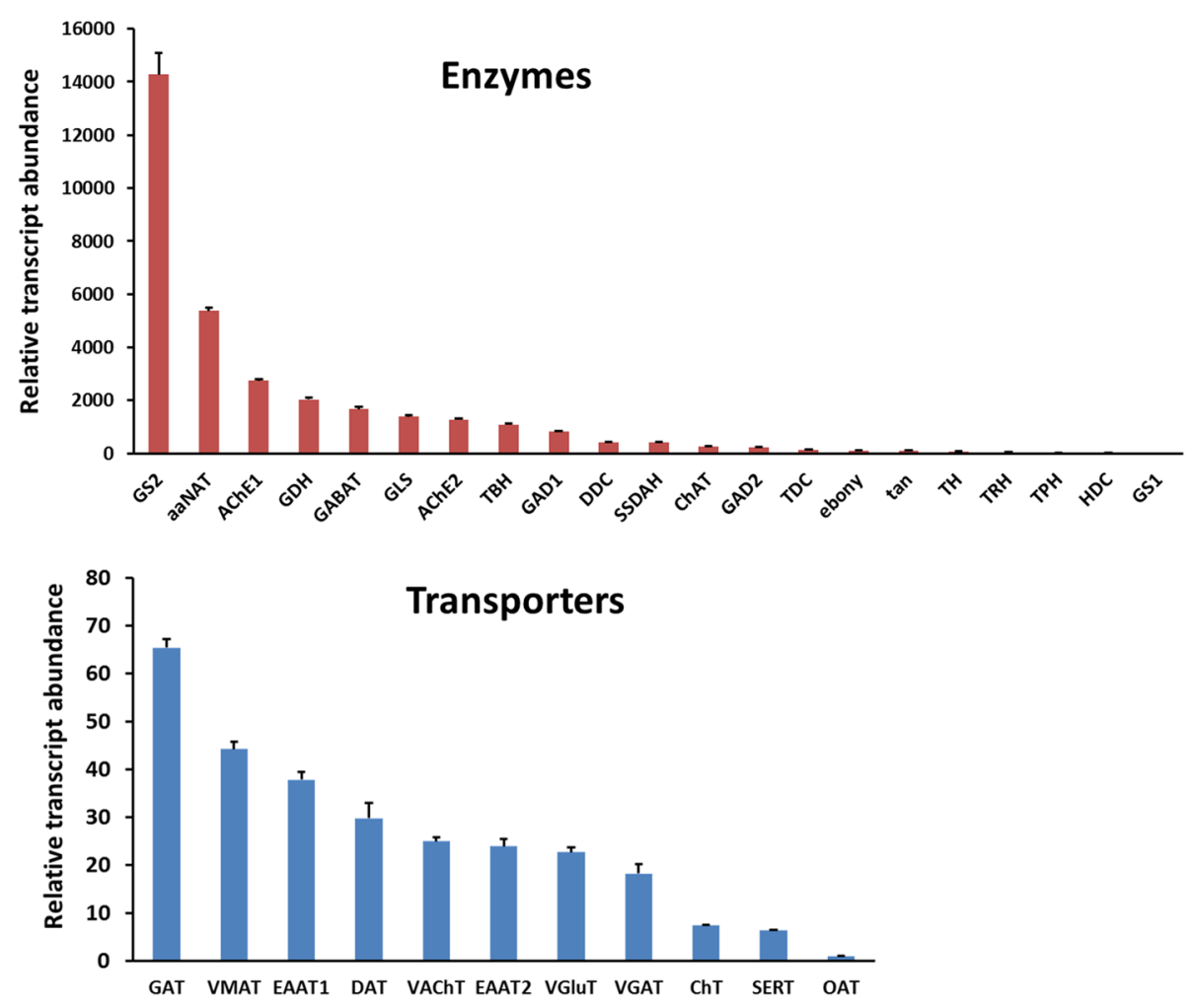

Fig. 8 qRT-PCR results showing the relative transcript abundance of the unigenes encoding enzymes and transporters involved in the neurotransmitter signaling systems in the central nervous system of $C$. suppressalis

photoreception in various arthropods, as well as in mechanoreception [54]. In arthropods, it was reported that histamine increases chloride conductance [55] and that its receptors are members of the ligand-gated chloride channel family. In $D$. melanogaster, histamine can modulate temperature preference behaviors [56] and regulate wake-promoting signals [57]. Immunohistochemical studies indicated the presence of histamine in a variety of neuron types in the brain and optic lobes, as well as in the ganglia of the ventral nerve cord of several insect species [58]. To produce histamine, the amino acid histidine is decarboxylated via a reaction catalyzed by histidine decarboxylase (HDC) [54] (Fig. 9d).

In order to clarify the molecular basis of the histaminergic signaling system, we identified histamine-related genes responsible for biosynthesis, signal transduction and reuptake in C. suppressalis central nervous system transcriptome. We only obtained one enzyme, HDC. Comparison of the deduced amino acid sequences of $C$. suppressalis HDC with other HDCs, indicated that Chilo HDC is most similar to the other known insect HDCs (89 \% identical to Danaus HDC, $68 \%$ to Apis HDC, $66 \%$ to Drosophila HDC, and 64\% to Tribolium HDC). The phylogenetic analysis of aromatic amino acid hydroxylases using the amino acid sequence of the Chilo HDC and various other aromatic amino acid hydroxylases also indicated that Chilo HDC is closely related to the insect HDC proteins (Fig. 5). The RT-PCR and qRT-PCR results demonstrated that HDC was highly expressed in the central nervous system (Figs. 10a and 11), indicating that the production of histamine is likely to occur mainly in the central nervous system. Unfortunately, the transporter that mediates histamine reuptake has not been fully characterized so far [59], but there is evidence that organic cation transporter (OCT) can function as a histamine transporter as well [60].

\section{Acetylcholinergic signaling}

Acetylcholine is the most abundant neurotransmitter in the brain of insects, particularly in the sensory pathways and specifically in the olfactory system. Acetylcholine is thought to be the major excitatory neurotransmitter in the central nervous system of insects as attested in honeybees, flies, grasshoppers, and locusts [61]. The acetylcholinergic system is one of the excitatory pathways participating in the parasympathicus, sympathicus, and the central nervous system using acetylcholine as a neurotransmitter [3]. Acetylcholine is synthesized from acetyl-CoA and choline via the action of the enzyme choline O-acetyltransferase (ChAT). Following the synthesis of acetylcholine, acetylcholine generated in neurons is antiported by protons into secretory vesicles 


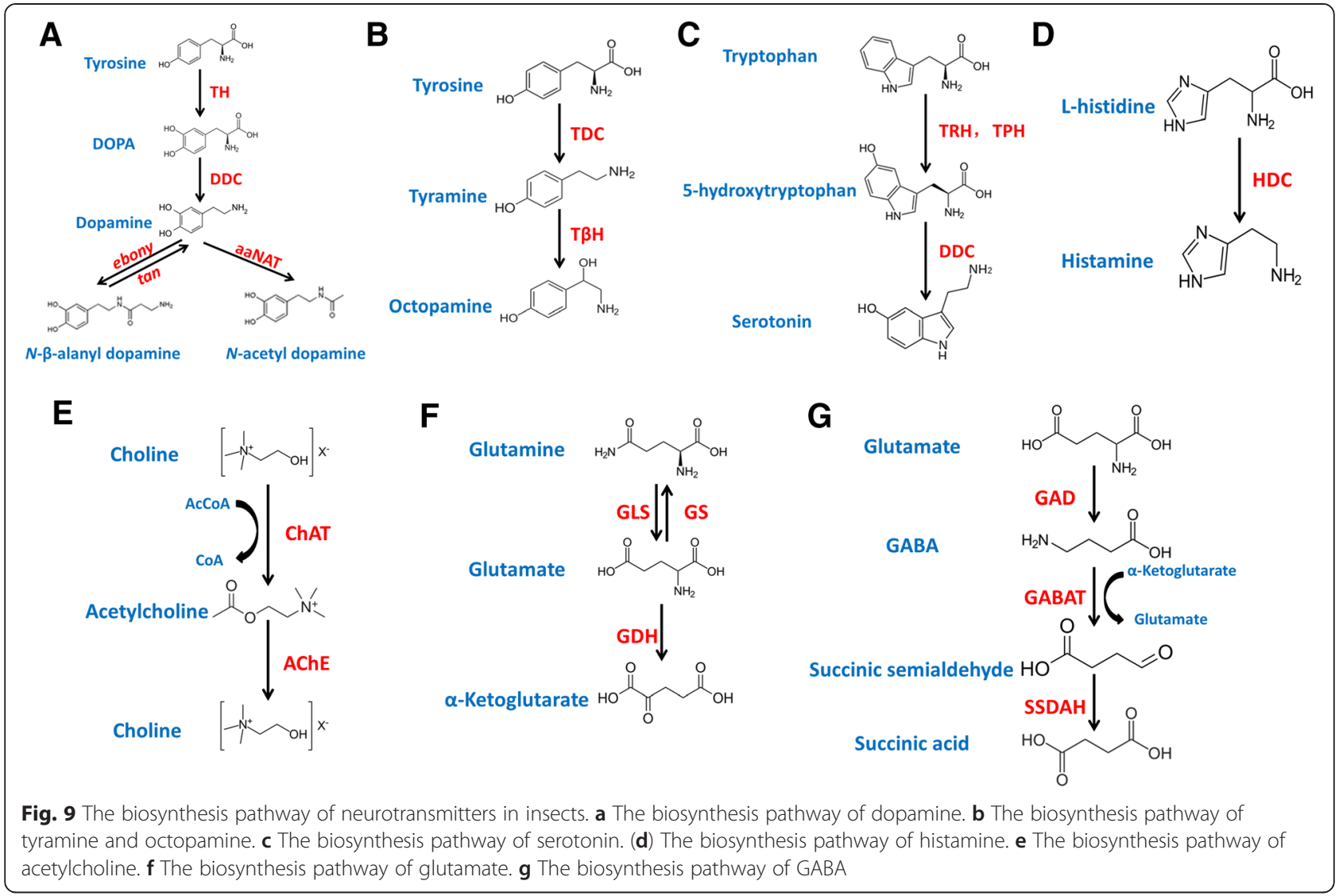

using vesicular acetylcholine transporter (VAChT). Upon its release from nerve terminal, triggered by an action potential, acetylcholine acts on its target tissues through two distinct receptor types, including nicotinic acetylcholine receptors (nAChRs) and muscarinic acetylcholine receptors (mAChRs) [62, 63]. Acetylcholine is a chemically stable compound that can persist for a long time after spreading into the synaptic cleft and spontaneous elimination is slow due to the quaternary ammonium atom in the choline moiety [3]. For this reason, the enzyme acetylcholinesterase (AChE) is just present in the synaptic cleft to quickly terminate the signal, and AChE hydrolyzes acetylcholine into acetic acid and choline [64]. While choline is transported from the synaptic cleft using choline transporter (ChT) back to the cytosol, the choline and acetic acid produced are then taken up and recycled by cholinergic neurons as precursors in new acetylcholine synthesis [65, 66] (Fig. 9e).

To elucidate the molecular basis of the acetylcholinergic signaling system, we identified acetylcholine-related genes involved in biosynthesis, signal transduction, and reuptake in C. suppressalis central nervous system transcriptome. We obtained three enzymes and two transporters, including ChAT, AChE1, AChE2, VAChT and ChT. Partial ChAT sequence containing 833 bp shows

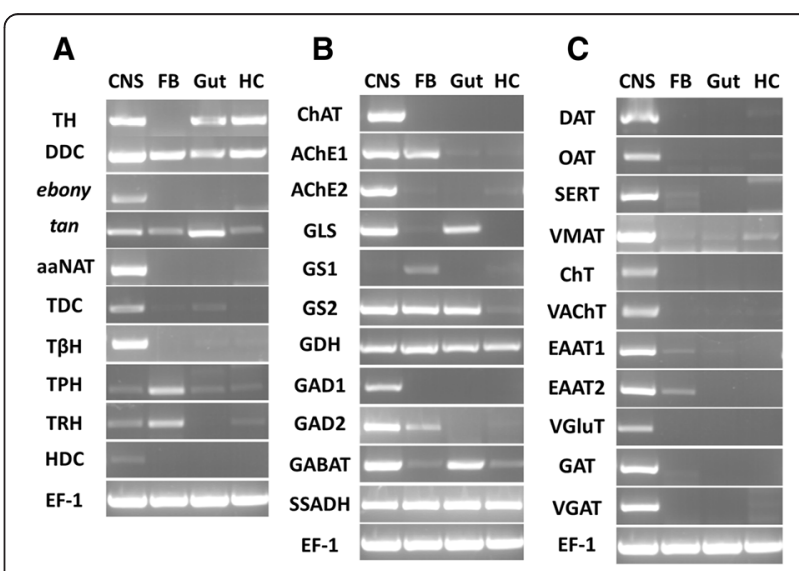

Fig. 10 RT-PCR results showing the relative expression of the $C$. suppressalis neurotransmitter-related genes in various tissues. a The relative expression of the enzymes involved in the biosynthesis pathway of biogenic amines in various tissues; b The relative expression of the enzymes involved in the biosynthesis pathway of acetylcholine, glutamate, and GABA in various tissues; $\mathbf{c}$ The relative expression of the transporters involved in the neurotransmitter signaling systems in various tissues. EF-1 was used as internal reference gene to test the integrity of each CDNA templates; the similar intensity of EF-1 bands between various tissues indicate the use of equal template concentrations. CNS, central nervous system; FB, fat body; Gut, gut; HC, hemocytes 

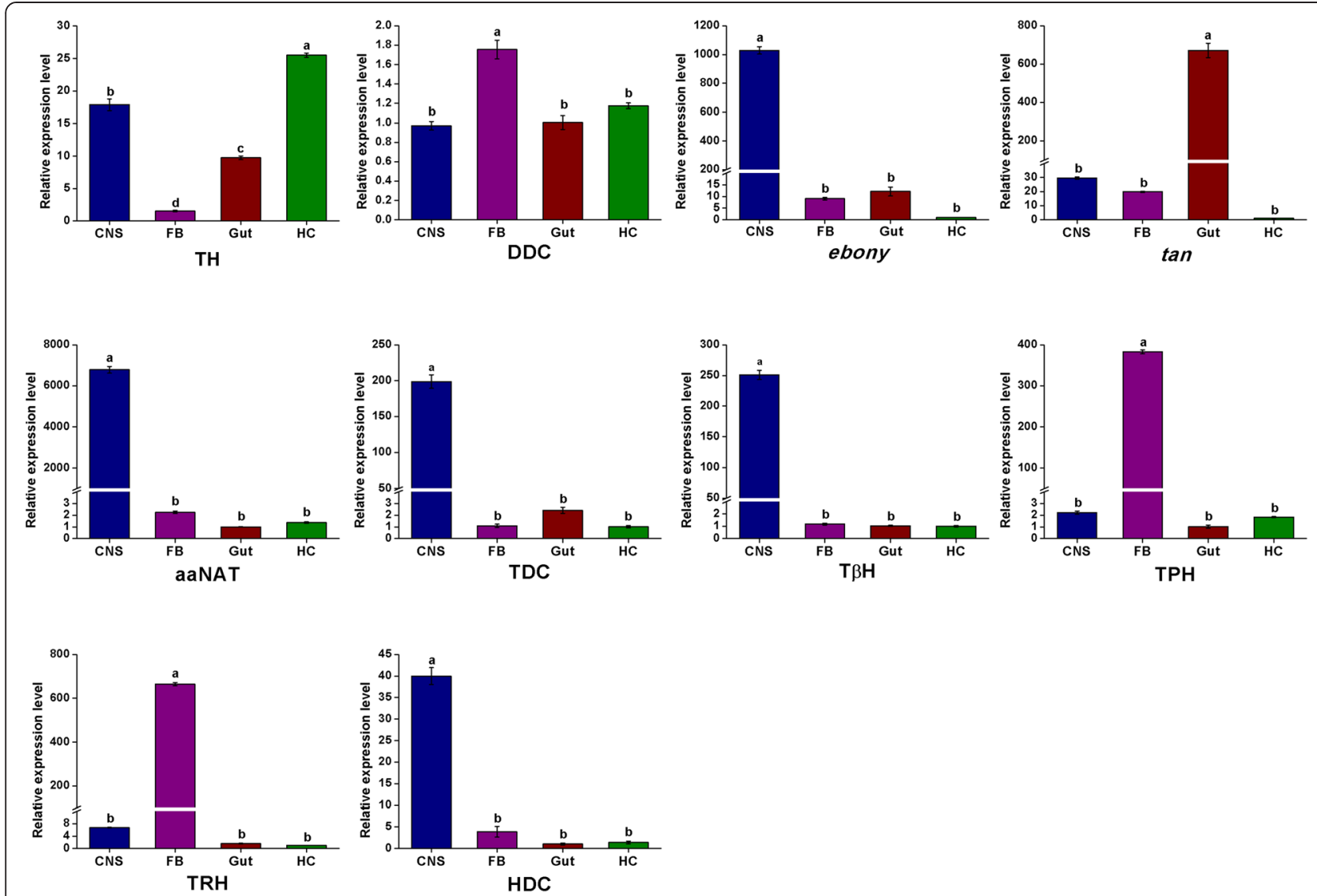

Fig. 11 qRT-PCR results showing the relative expression levels of the enzymes involved in the biosynthesis pathway of biogenic amines in various tissues in C. suppressalis. The standard error is represented by the error bar, and the different letters above each bar denote significant differences $(p<0.05)$

$83 \%$ identity in amino acid sequence with ChAT of $B$. mori (Protein ID: BAO23491.1). Two unigenes encoding AChE1 and AChE2 in C. suppressalis transcriptome show $100 \%$ identity in amino acid sequences with the published AChE1 and AChE2 of C. suppressalis (Protein ID: ABO38111.1 and ABR24230.1, respectively) (Table 2). Multiple sequence alignment of acetylcholinesterases revealed that choline binding sites and active site triad residues were conserved in AChEs (Additional file 7). AChEs have been specialized as the main catalytic enzymes and become very effective targets for both organophosphorus and carbamate insecticides in different insect species [67-69]. In addition, two transporters ChT and VAChT show $93 \%$ and $87 \%$ identity in amino acid sequences with ChT of Trichoplusia ni (Protein ID: AAT88074.1) and VAChT of B. mori (Protein ID: NP_001275599.1), respectively (Table 3). The RT-PCR and qRT-PCR results revealed that ChAT, AChE1 and AChE2 were all highly expressed in the central nervous system (Figs. 10b and 12). ChAT with higher activity is localized in the cytosol near neurosynapses as a soluble molecule, and ChAT can also be found as a membrane bound protein [65]. AChEs are essential enzymes at the synapses of cholinergic neurons in the central and peripheral nervous systems, to catalyze the hydrolysis of the neurotransmitter acetylcholine, thus terminating neurotransmission [70]. Additionally, the RT-PCR and qRT-PCR results revealed that $\mathrm{ChT}$ and VAChT were significantly expressed in the central nervous system (Fig. 10c and 13), indicating that the two transporters may play a major role in neurotransmitter uptake and neurotransmission. Interestingly, acetylcholine and acetylcholine receptors are known to be present on many cell types, including endothelial cells and cells of the immune system, suggesting that there exists a connection between the immune system and the nervous system [71].

\section{Glutamatergic signaling}

Glutamate is a major neurotransmitter in both vertebrates and invertebrates. Glutamate acts as an excitatory neurotransmitter in vertebrates, whereas it functions as both an excitatory and an inhibitory neurotransmitter in 
invertebrates. Two closely related amino acids, $\gamma$ aminobutyric acid and glutamate, play an important role as the major inhibitory neurotransmitters in invertebrates. Glutamatergic inhibitory neurotransmission is mediated by pentameric glutamate-gated chloride channels in the invertebrate nervous system [72]. In arthropods, glutamate participates in regulating multiple physiological processes such as neuron sensibility modulation [73], juvenile hormone synthesis [74], control of rest/arousal neurons [75], olfactory memory [76], locomotion and feeding [77]. Glutamate is synthesized from glutamine via the action of the enzyme glutaminase (GLS). Within neurons, packaging of this neurotransmitter is achieved via vesicular glutamate transporter (VGluT). Glutamate released from synaptic vesicles acts on target tissues via a number of glutamate receptors. Following its release, the actions of glutamate are terminated by its reuptake via excitatory amino acid transporters (EAATs) located primarily in glia, and its subsequent conversion back to glutamine via the action of glutamine synthetase (GS), or degradation into $\alpha$ - ketoglutarate via the action of glutamate dehydrogenase (GDH) [1] (Fig. 9f).

To illustrate the molecular basis of the glutamatergic signaling system, glutamate-related genes involved in biosynthesis, signal transduction, and reuptake were found in C. suppressalis central nervous system transcriptome. We found four enzymes and three transporters, including GLS, GS1, GS2, GDH, VGluT, EAAT1 and EAAT2. Comparison of C. suppressalis GLS (643 amino acids) with D. plexippus GLS (Protein ID: EHJ71111.1) revealed $87 \%$ identity between the two proteins. Two unigenes encoding GS1 and GS2 in C. suppressalis transcriptome show $75 \%$ and $93 \%$ identities amino acid sequences with GS1 of B.mori (Protein ID: XP_004930366.1) and GS2 of P. xuthus (Protein ID: BAM17922.1), respectively (Table 2). Amino acid sequence alignment of glutamine synthetases suggested that the residues involved in binding of glutamate, ATP, and ammonia were conserved in GSs (Additional file 8). GDH contains an open reading frame of 1,665 bp with $95 \%$ identity in amino acid sequence to GDH of Papilio

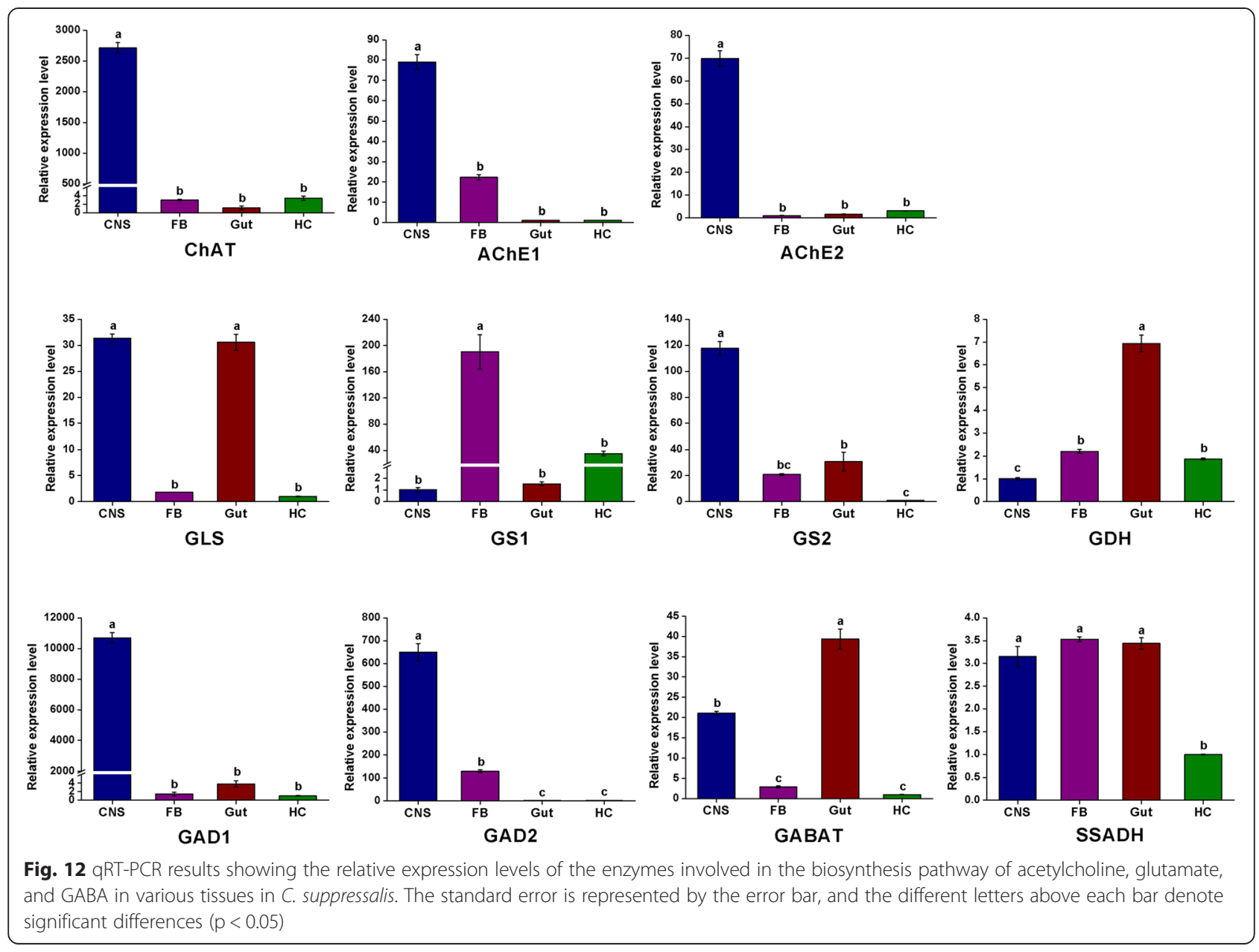




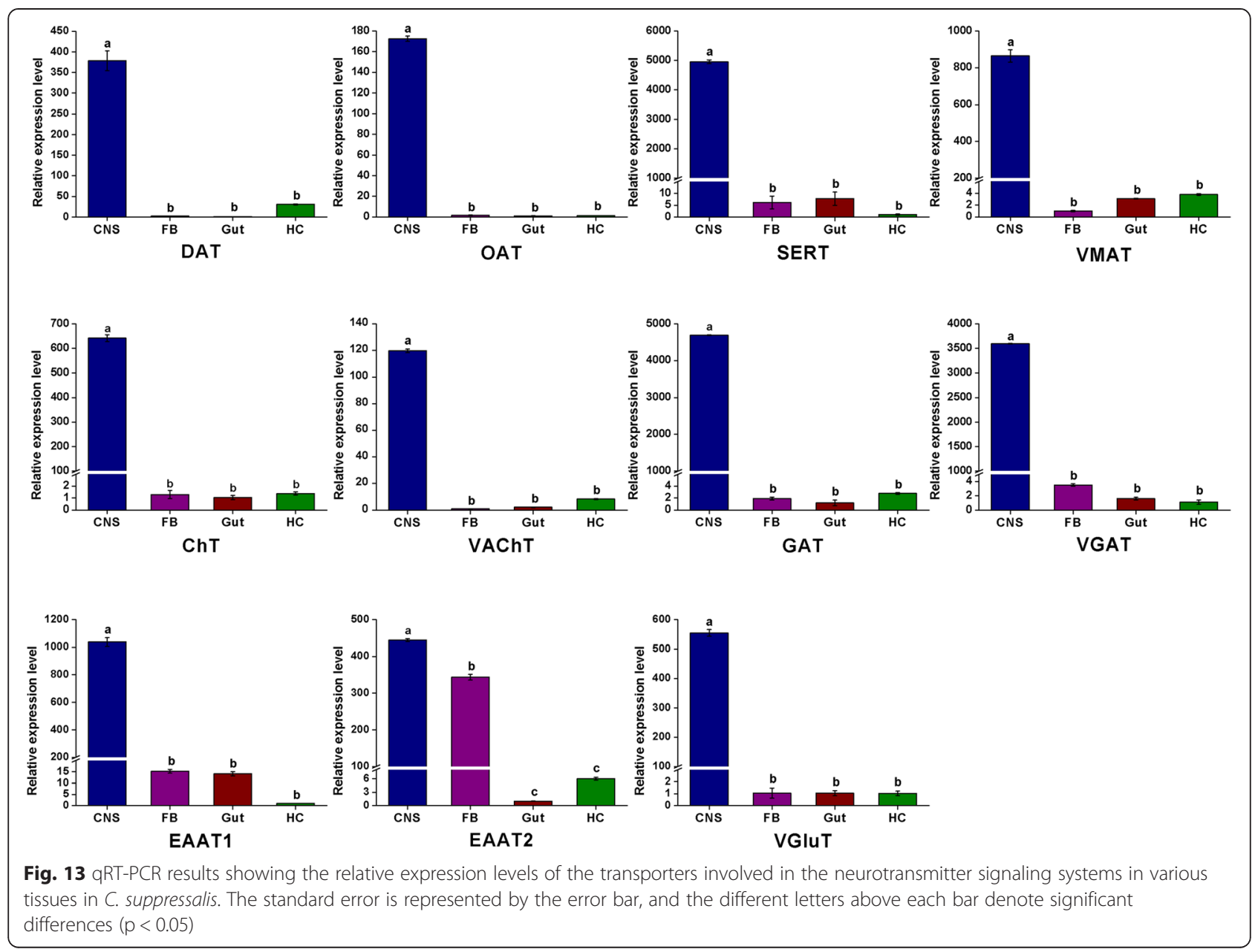

polytes (Protein ID: BAM20330.1) (Table 2). VGluT with an open reading frame of 1,782 bp shows $85 \%$ identity in amino acid sequence to B.mori VGluT (Protein ID: XP_004925576.1). Moreover, two excitatory amino acid transporters function as glutamate transporters, two unigenes encoding EAAT1 and EAAT2 show $86 \%$ and $83 \%$ identities in amino acid sequences with EAAT1 of $T . n i$ (Protein ID: AAB84380.1) and EAAT2 of B.mori (Protein ID: NP_001240825.1), respectively (Table 3). Multiple sequence alignment of excitatory amino acid transporters indicated that the amino acid sequences of the two types of EAATs were conserved (Additional file 9). The RT-PCR and qRT-PCR results revealed that GLS was highly expressed in the central nervous system and gut, GS1 was highly expressed in fat body, GS2 was highly expressed in the central nervous system, while GDH was highly expressed in gut (Figs. 10b and 12). In addition, the RTPCR and qRT-PCR results revealed that VGluT and EAAT1 were specifically expressed in the central nervous system, whereas EAAT2 was highly expressed in both central nervous system and fat body (Figs. 10c and 13). In $D$. melanogaster, EAAT1 was expressed in a population of neurons, located between the lamina and medulla neuropils of the optic lobes, to address glial processes that closely follow the motor axons up to the neuromuscular junction. EAAT2 was expressed in the central and peripheral nervous systems to regulate selective olfactory and gustatory functions [78].

\section{GABAergic signaling}

Neurons communicate with each other via signaling molecules. In vertebrate fast neurotransmission, $\gamma$ aminobutyric acid (GABA) acts as an inhibitory neurotransmitter. The precursor of GABA is glutamate. It is interesting that these two closely related amino acids share an inhibitory neurotransmitter role in invertebrates [79]. For insects, GABA is reported to modulate various physiological behaviors such as copulation persistence [28], feeding restraint [80], locomotion [81], sleep [82], circadian clock [83], response to alcohol [84], and olfactory memory [85]. GABA is synthesized from glutamate via the actions 
of the enzyme glutamic acid decarboxylase (GAD). Once synthesized, vesicular GABA transporter (VGAT) packages GABA into synaptic vesicles. GABA is released into synaptic cleft from the presynaptic neurons by depolarization triggered by the action potential. The released GABA traverses the synaptic cleft and binds to ionotropic receptors localized in the postsynaptic membrane to provoke an electrical change in the postsynaptic neurons. GABA also activates GPCRs to elicit intracellular signal transduction. Then, the actions of GABA are terminated by its reuptake via GABA transporter (GAT) and its subsequent conversion back to glutamate via the action of GABA transaminase (GABAT), or degradation via the enzyme succinic semialdehyde dehydrogenase (SSADH) [1, 86, 87] (Fig. 9g).

In C. suppressalis central nervous system transcriptome, GABA-related genes responsible for biosynthesis, signal transduction, and reuptake were identified. We found GAD1, GAD2, GABAT, SSADH, VGAT and GAT. GABA is synthesized by two isoforms of the pyridoxal 5'-phosphate-dependent enzyme glutamic acid decarboxylase (GAD1 and GAD2) [88]. Two unigenes encoding GAD1 and GAD2 in C. suppressalis transcriptome show $83 \%$ and $86 \%$ identities in amino acid sequences with GAD1 of B.mori (Protein ID: XP_004925034.1) and GAD2 of Biston betularia (Protein ID: AEP43793.2), respectively (Table 2). Multiple sequence alignment of glutamate decarboxylase showed that the proposed substrate binding domain, the 'decarboxylation' domain, active site residues, and functional residues were conserved in GADs (Additional file 10). GABAT with an open reading frame of 1,482 bp shows $74 \%$ identity in amino acid sequence to $D$. plexippus GABAT (Protein ID: EHJ72994.1). SSADH contains an open reading frame of 1,521 bp with $77 \%$ identity in amino acid sequence to SSADH of B.mori (Protein ID: XP_004932642.1) (Table 2). In addition, the two transporters VGAT and GAT show $88 \%$ and $96 \%$ identities in amino acid sequences with VGAT of D. plexippus (Protein ID: AAT88074.1) and GAT of T. $n i$ (Protein ID: AAF70819.1), respectively (Table 3). The RT-PCR and qRT-PCR results showed that both GAD1 and GAD2 were specifically expressed in the central nervous system, GABAT was highly expressed in the central nervous system and gut, while SSADH was not significantly different at expression levels in various tissues (Figs. 10b and 12). Furthermore, the two transporters VGAT and GAT were significantly expressed in the central nervous system (Figs. 10c and 13).

\section{Conclusions}

The rice striped stem borer C. suppressalis is a destructive rice pest in China. In recent years, the damage caused by C. suppressalis has increased dramatically in China and has posed a severe threat to high and stable crop yields through changes in the rice cultivation system and the popularization of hybrid rice. To date, control of this insect still relies mainly on chemical pesticides, which has led to the development of resistance to organophosphate and nereistoxin insecticides due to excessive use in the field $[7,13]$. Our study provides information and resource to identify and facilitate functional studies of genes responsible for neurotransmitter biosynthesis, transport and degradation at the molecular level. By do novo sequencing of the $C$. suppressalis central nervous system transcriptome, we obtained 54,411 assembled unigenes. Among these unigenes, we have identified 32 unigenes encoding 21 enzymes and 11 transporters putatively involved in neurotransmitter signaling systems by local blast. We further confirmed these unigenes via homology search and phylogenetic tree analysis (Tables 2, 3 and Figs. 5, 6 and 7). For each neurotransmitter, we searched for and identified proteins involved in its biosynthesis, packaging, and recycling/degradation. We revealed the expression profiles of enzymes and transporters in various tissues by RT-PCR and qRT-PCR, which indicated that most enzymes were highly expressed in the central nervous system and all the transporters were specifically expressed in the central nervous system. In addition, the transcript abundances of enzymes and transporters in the central nervous system were validated by qRT-PCR (Fig. 8). The high expression levels may reflect their important roles in the central nervous system. Interestingly, the expression of enzymes and transporters also could be detected in hemocytes, these results were consistent with the previous studies that the neurotransmitters may be important molecules bridging the nervous system and immune system $[3,37$, $44,51]$. In this study, we summarized how the neurotransmitters are produced, released and recycled between presynaptic terminal and synaptic cleft, and how the enzymes and transporters function in the neurotransmitter signaling systems (Fig. 14). To our knowledge, this is the first study to characterize neurotransmitter signaling pathways comprehensively in rice pest. It is known that the enzymes are prime targets of pesticides because of their important roles in biosynthesis and degradation of the neurotransmitters. In addition, due to their crucial roles in insect nutrition and phylogenetic specificity, neurotransmitter transporters are considered to be excellent targets for the development of lineage-specific and environmentally safe insecticides [89]. Therefore, our study provides a valuable resource of molecular information for future investigations of the functions of neurotransmitterrelated genes and developing new potential pesticides for insect pest control. 


\section{Methods}

\section{Insect rearing}

The C. suppressalis colony has been reared in our laboratory continuously, of which larvae were originally collected from the rice field in Fuyang, Zhejiang Province, China, in 2012. The larvae were reared on artificial diet [90] and kept at $25 \pm 1{ }^{\circ} \mathrm{C}$ with approximately $80 \%$ relative humidity under a 14:10 light:dark cycle.

\section{Sample collection and RNA isolation}

The fifth instar larvae of C. suppressalis were surfacesterilized with $75 \%$ ethanol. Then, the central nervous system (brain, suboesophageal ganglion, thoracic ganglion and abdominal ganglion) samples were individually dissected under saline solution with RNase inhibitor (TaKaRa, Japan) from 100 larvae. Total RNA samples were extracted using TRIzol Reagent (Invitrogen, Carlsbad, CA, USA) following the manufacturer's instructions and stored in $-80{ }^{\circ} \mathrm{C}$. RNA concentration was measured using Qubit ${ }^{\oplus}$ RNA Assay Kit in Qubit ${ }^{\circ}$ 2.0 Flurometer (Life Technologies, CA, USA). RNA integrity was assessed using the RNA Nano 6000 Assay Kit of the Bioanalyzer 2100 system (Agilent Technologies, CA, USA).

\section{cDNA library construction}

A total amount of $3 \mu \mathrm{g}$ RNA per sample was used for construction of cDNA library. All samples had RIN (RNA
Integrity Number) values above 8 . Sequencing of libraries was performed using Illumina TruSeq ${ }^{\mathrm{TM}}$ RNA Sample Preparation Kit (Illumia, San Diego, USA) following manufacturer's recommendations and four index codes were added to attribute sequences to each sample. Briefly, mRNA was purified from total RNA using poly-T oligoattached magnetic beads. Fragmentation was carried out using divalent cations under elevated temperature in Illumina proprietary fragmentation buffer. First strand cDNA was synthesized using random oligonucleotides and SuperScript II. Second strand cDNA synthesis was subsequently performed using DNA polymerase I and RNase $\mathrm{H}$. Remaining overhangs were converted into blunt ends via exonuclease/polymerase activities and enzymes were removed. After adenylation of 3' ends of DNA fragments, Illumina PE adapter oligonucleotides were ligated for hybridization. In order to select cDNA fragments of preferentially 200 bp in length, the library fragments were purified with AMPure XP system (Beckman Coulter, Beverly, USA). DNA fragments with ligated adaptor molecules on both ends were selectively enriched using Illumina PCR Primer Cocktail in a 10 cycle PCR reaction. Products were purified (AMPure XP system) and quantified using the Agilent high sensitivity DNA assay on the Agilent Bioanalyzer 2100 system (Agilent Technologies, CA, USA) [91].

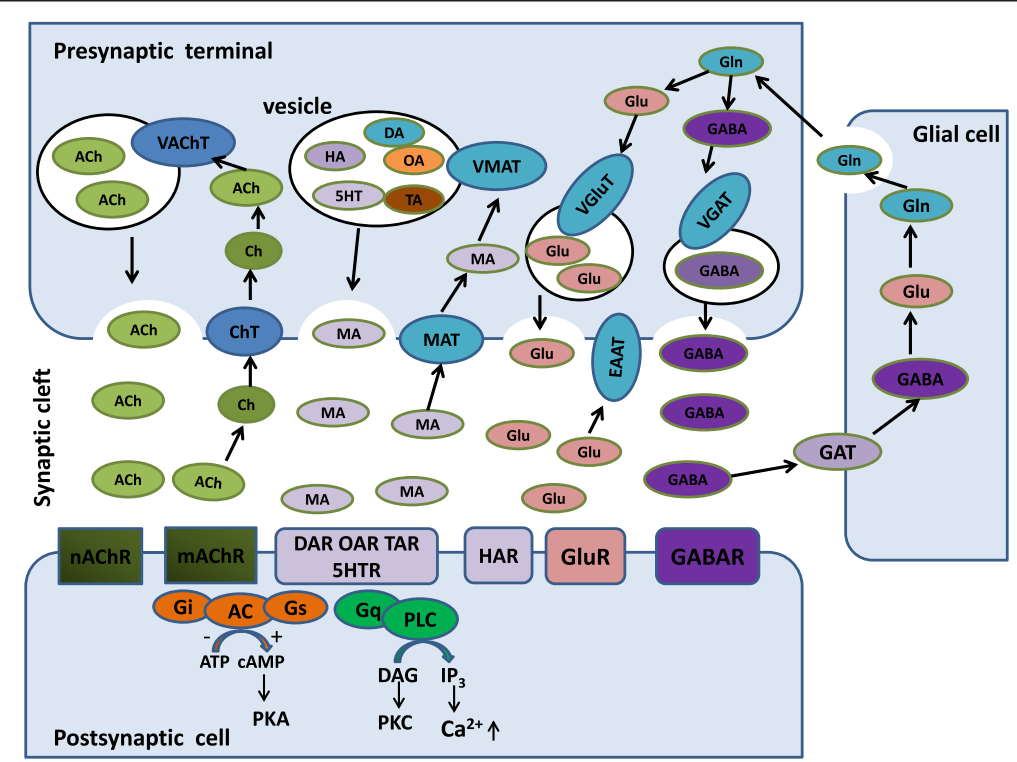

Fig. 14 Putative neurotransmitter signaling pathways in insects. ACh, acetylcholine; Ch, choline; ChT, choline transporter; VAChT, vesicular acetylcholine transporter; $\mathrm{nAChR}$, nicotinic acetylcholine receptor; $\mathrm{mAChR}$, muscarinic acetylcholine receptor; DA, dopamine; OA, octopamine; TA, tyramine; $5 \mathrm{HT}$, serotonin; HA, histamine; MA, monoamine; MAT, monoamine transporter; VMAT, vesicular monoamine transporter; DAR, dopamine receptor; OAR, octopamine receptor; TAR, tyramine receptor; 5HTR, serotonin receptor; HAR, histamine receptor; Glu, glutamate; Gln, glutamine; EAAT, excitatory amino acid transporter; VGluT, vesicular glutamate transporter; GluR, glutamate receptor; GABA, $ү$-aminobutyric acid; GAT, GABA transporter; VGAT, vesicular GABA transporter; GABAR, GABA receptor; AC, adenylyl cyclase; DAG, diacylglycerol; IP3, 1,4,5-trisphosphate; PKA, protein kinase A; PKC: protein kinase C; PLC, phospholipase C; ATP, adenosine triphosphate; CAMP, cyclic adenosine monophosphate 
Illumina sequencing, assembly, and annotation

Transcriptome sequencing was carried out on an Illumina HiSeq 2000 platform that generated about 100 bp paired-end (PE100) raw reads (Novogene Bioinformatics Technology Co.Ltd). Raw data were deposited to NCBI Short Read Archive (SRA) database (http://www.ncbi.nlm.nih.gov/Traces/sra/). After removing adaptor sequences, ambiguous ' $\mathrm{N}$ ' nucleotides (with the ratio of ' $\mathrm{N}$ ' to be more than $10 \%$ ) and low quality sequences (with quality score to be less than 5), the remaining clean reads were assembled using Trinity software as described for de novo transcriptome assembly without reference genome. For homology annotation, non-redundant sequences were subjected to public databases, including NCBI (http://www.ncbi.nlm.nih.gov/) non-redundant protein $(\mathrm{Nr})$ and non-redundant nucleotide (Nt), SwissProt (http://www.ebi.ac.uk/uniprot/), Gene Ontology (GO) (http://www.geneontology.org/), Clusters of Orthologous Groups (COG) (http://www.ncbi.nlm.nih.gov/COG/), and Kyoto Encyclopedia of Genes and Genomes (KEGG) (http://www.genome.jp/kegg/). If results of different databases were conflicted, a priority order of alignments from Nr, Nt, KEGG, SwissProt, GO and COG databases was followed. Comparing to $\mathrm{Nr}$, Nt and SwissProt databases were carried out using BLASTX algorithm with an E-value cut-off of $10^{-10} \mathrm{GO}$ terms at $2^{\text {nd }}$ level was used to perform GO annotation. COG and KEGG classifications were done using BLASTX with an E-value cut off of $10^{-5}$

\section{Identification of putative genes associated with neurotransmitter biosynthesis and transport}

We used amino acid sequences of enzymes and transporters from the fruit fly D. melanogaster and other invertebrates that are associated with neurotransmitter biosynthesis and transport as queries for BLAST analysis (TBLASTN) to search the candidate sequences of enzymes and transporters in C. suppressalis. The BLAST + 2.2.23 software (downloadable from the National Center for Biotechnology Information, Bethesda, MD, USA; ftp://ftp.ncbi.nlm.nih.gov/blast/executables/blast $+/$ ) was used for local BLAST to search the assembled unigenes. After the identification of genes, we performed BLASTX and BLASTN programs against non-redundant protein $(\mathrm{Nr})$ and nucleotide sequence (Nt) databases at NCBI to find the homologous sequences in other insects [92].

\section{Phylogenetic analysis and sequence alignment}

To identify potential orthologs of the genes associated with neurotransmitter biosynthesis and transport, we constructed the phylogenetic trees of these putative genes in C. suppressalis and other insects. The sequences were aligned using ClustalW2 (http://www.ebi.ac.uk/ Tools/ msa/clustalw2/). The tree was drawn using MEGA 5.0 with the maximum likelihood method [93] and the branch support values are expressed as percentages. Multiple sequence alignments of the complete amino acid sequences were performed with ClustalX2 [94] and edited with software GeneDoc. The accession numbers of sequences used in this study were shown in Additional file 11.

\section{RT-PCR and qRT-PCR}

The total RNA were isolated from fifth instar larval central nervous system, gut (foregut, midgut, hindgut, and Malpighian tube), hemocytes and fat body. cDNA was synthesized from $1 \mu \mathrm{g}$ RNA using TransScript OneStep gDNA Removal and cDNA Synthesis SuperMix (Transgen, Beijing, China) for RT-PCR and qRT-PCR. Specific primers for RT-PCR and qRT-PCR analysis were designed with Primer 3 (http://bioinfo.ut.ee/primer3-0.4.0/) (Additional files 12 and 13). RT-PCR was carried out in a $50 \mu \mathrm{l}$ reaction containing $5 \mu \mathrm{l} 10 \times$ TaKaRa Ex Taq, $4 \mu \mathrm{l}$ dNTP Mixture, $0.5 \mu \mathrm{l}$ TaKaRa Ex Taq (TaKaRa, Japan), $2 \mu$ l each primer $(10 \mu \mathrm{M})$, $1 \mu \mathrm{l}$ cDNA template, $35.5 \mu \mathrm{l}$ sterile $\mathrm{H}_{2} \mathrm{O}$. The PCR cycling profile was: $94{ }^{\circ} \mathrm{C}$ for $3 \mathrm{~min}$, followed by 40 cycles of $94{ }^{\circ} \mathrm{C}$ for $30 \mathrm{sec}, 60{ }^{\circ} \mathrm{C}$ for $30 \mathrm{sec}, 72{ }^{\circ} \mathrm{C}$ for $1 \mathrm{~min}$ and a final extension for $10 \mathrm{~min}$ at $72{ }^{\circ} \mathrm{C}$. PCR products were separated in $1.5 \%$ agarose gels and stained with ethidium bromide. qRT-PCR was conducted using the CFX Connect ${ }^{\mathrm{Tm}}$ Real-Time Detection System (Bio-rad, USA). The reference gene, elongation factor 1 alpha (EF-1), was used for normalizing expression of the target gene. qRT-PCR was done in a $25 \mu$ l reaction containing $12.5 \mu \mathrm{l} \mathrm{SYBR}^{\odot}$ Premix Ex $\mathrm{Taq}^{\mathrm{TM}}$ II (Tli RNaseH Plus) (TaKaRa, Japan), $1 \mu \mathrm{l}$ each primer $(10 \mu \mathrm{M}), 5 \mu \mathrm{l}$ cDNA template, $5.5 \mu$ sterile $\mathrm{H}_{2} \mathrm{O}$. The qRT-PCR procedure was $95{ }^{\circ} \mathrm{C}$ for $30 \mathrm{sec}$, followed by 40 cycles of $95{ }^{\circ} \mathrm{C}$ for $5 \mathrm{sec}$ and $60{ }^{\circ} \mathrm{C}$ for $30 \mathrm{sec}$. Then, the PCR products were heated to $95{ }^{\circ} \mathrm{C}$ for $15 \mathrm{sec}$, cooled to $60{ }^{\circ} \mathrm{C}$ for $1 \mathrm{~min}$ and heated to $95{ }^{\circ} \mathrm{C}$ for $30 \mathrm{sec}$ and cooled to $60{ }^{\circ} \mathrm{C}$ for $15 \mathrm{sec}$ to measure the dissociation curves. Three biological samples of each tissue were used to ensure the reliability and reproducibility.

\section{qRT-PCR data analysis}

The relative quantification in each tissue was calculated using the comparative $2^{-\Delta \Delta C T}$ method [95]. All data were normalized to endogenous elongation factor 1 alpha level from the same individual samples. In the analysis of the relative expression level in different tissues, the lowest expression level was taken as the calibrator. Thus, the relative expression level in different tissues was assessed by comparing the expression level of each target gene in other tissues to that in the lowest part. The results are presented as the mean of the expression level 
in three biological replicates. The data of relative expression levels in various tissues were analyzed using oneway analysis of variance (ANOVA), followed by a Tukey's honestly significant difference (HSD) test when significant differences were tested. All statistical analysis was performed by Data Processing System (DPS) package (Version 9.5) [96].

\section{Availability of supporting data}

The sequences of the unigenes used in the study have been submitted to the NCBI. The transcriptomic data of $C$. suppressalis central nervous system has been submitted to Sequence Read Archive (SRA) database (http://www.ncbi.nlm.nih.gov/sra), and the accession number is SRR2015503.

\section{Additional files}

Additional file 1: Summary of distribution of assembled length in C. suppressalis central nervous system transcriptome.

Additional file 2: The identified unigenes with putative roles in C. suppressalis neurotransmitters biosynthesis and transport (Table 2, 3).

Additional file 3: Gene ontology classification of the C. suppressalis central nervous system transcriptome.

Additional file 4: COG functional classification of the $C$. suppressalis central nervous system transcriptome.

Additional file 5: KEGG functional classification of the $C$. suppressalis central nervous system transcriptome. A, Cellular Processes; B, Environmental Information Processing; C, Genetic Information Processing; D, Metabolism; E, Organismal Systems.

Additional file 6: Amino acid sequence alignment of tyrosine hydroxylase homologues. The sequences are from CsTH-L (KP657623), CsTH-S (KP657624), MsTH-L (BAF32573.1), MsTH-S (BAF32574.1), DmTH-L (NP_476898.1) and DmTH-S (NP_476897.1). DmTH (Ser32) is phosphorylated by CAMP-dependent protein kinase and is conserved in CsTH (Ser31) (red asterisk). The putative catalytic domain is indicated between two red dotted lines. Alternatively spliced domains are marked with red arrows.

Additional file 7: Amino acid sequence alignment of acetylcholinesterase homologues. The sequences are from DmAChE (P07140.1), CSAChE1 (KP657634), BmAChE1 (ABY50088.1), TCAChE1 (ADU33189.1), CsAChE2 (KP657635), BmAChE2 (ABY50089.1), and TCAChE2 (ADU33190.1). The number 1, 2, 3 on the amino acids show the residues forming intramolecular disulfide bonds. The active site triad residues are marked with red filled diamonds. Purple filled triangles indicate the oxyanion hole-forming residues. Black filled circles represent the acylpocket, while open green diamonds mark the peripheral anionic site. The choline binding site is indicated by the blue arrows. The cholinesterase signature sequence is underlined.

Additional file 8: Amino acid sequence alignment of glutamine synthetase homologues. The sequences are from CsGS1 (KP657637), BmGS1 (XP_004930366.1), DmGS1 (NP_476570.1), CsGS2 (KP657638), BmGS2 (XP_004929856.1), and DmGS2 (NP_511123.2). Residues involved in binding of glutamate (red filled triangles), ATP (purple filled diamonds), and ammonia (black filled circles) are highlighted.

Additional file 9: Amino acid sequence alignment of excitatory amino acid transporter homologues. The sequences are from CSEAAT1 (KP657650), BmEAAT1 (NP_001240824.1), DmEAAT1 (NP_477428.1), CsEAAT2 (KP657651), BmEAAT2 (NP 001240825.1), DmEAAT2 (NP_001162844.1).
Additional file 10: Amino acid sequence alignment of glutamate decarboxylase homologues. The sequences are from CsGAD1 (KP657640), BmGAD1 (XP_004925034.1), DmGAD1 (NP_523914.2), CsGAD2 (KP657641), BmGAD2 (XP_004932908.1), and DmGAD2 (NP_001285910.1). Domain "a" is the proposed substrate binding domain. Domain 'b' is the 'decarboxylation' domain and contains the pyridoxal binding site 'NPHK'(underlined). The conserved active site residues are marked with purple arrows. The functional residues are indicated by red filled triangles. Additional file 11: The accession number of the sequences used in this study.

Additional file 12: Primers used for RT-PCR analysis of enzyme and transporter genes in C. suppressalis.

Additional file 13: Primers used for qRT-PCR analysis of expression levels of enzyme and transporter genes in C. suppressalis.

\section{Abbreviations}

DA: dopamine; OA: octopamine; TA: tyramine; $5 \mathrm{HT}$ : serotonin; HA: histamine; MA: monoamine; MAT: monoamine transporter; DAT: dopamine transporter; OAT: octopamine transporter; SERT: serotonin transporter; VMAT: vesicular monoamine transporter; DAR: dopamine receptor; OAR: octopamine receptor; TAR: tyramine receptor; 5 HTR: serotonin receptor; HAR: histamine receptor; TH: tyrosine hydroxylase; DDC: dopa decarboxylase; TDC: tyrosine decarboxylase; TBH: tyramine beta hydroxylase; HDC: histidine decarboxylase; TRH: tryptophan hydroxylase; TPH: phenylalanine hydroxylase; ebony: NBAD synthetase; tan: NBAD hydrolase; aaNAT: arylalkylamine-N-acetyl transferase; ACh: acetylcholine; Ch: choline; ChT: choline transporter; VAChT: vesicular acetylcholine transporter; nAChR: nicotinic acetylcholine receptor; mAChR: muscarinic acetylcholine receptor; AChE: acetylcholinesterase; ChAT: choline acetyltransferase; Glu: glutamate; Gln: glutamine; EAAT: excitatory amino acid transporter; VGluT: vesicular glutamate transporter; GluR: glutamate receptor; GDH: glutamate dehydrogenase; GLS: glutaminase; GS: glutamine synthetase; GABA: $y$-aminobutyric acid; GAT: GABA transporter; VGAT: vesicular GABA transporter; GABAR: GABA receptor; GAD: glutamic acid decarboxylase; GABAT: GABA transaminase; SSADH: succinic semialdehyde dehydrogenas; AC: adenylyl cyclase; DAG: diacylglycerol; IP3: 1,4,5-trisphosphate; PKA: protein kinase A; PKC: protein kinase C; PLC: phospholipase C; ATP: adenosine triphosphate; CAMP: cyclic adenosine monophosphate.

\section{Competing interests}

The authors declare that they have no competing interests.

\section{Authors' contributions}

GX, SFW, QF, and GYY conceived and designed the experimental plan. GX, GXG and YSW preformed the experiments. GX analyzed and interpreted the sequence data and experimental data. GX, SFW, and GYY drafted the manuscript. All authors read and approved the final manuscript.

\section{Acknowledgements}

This work was supported by National High Technology Research and Development Program of China (863 Program, 2011AA10A204), National Special Agricultural Research Projects for Public Welfare, China (201303017) and National Science Fund for Innovative Research Groups of Biological Control (Grant 31321063). The authors sincerely thank Zi-Wen Teng, Shuang-Yang Wu, Pi-Hua Zhou, Fang Liu, Lu-Lu Gu, and Gui-Xiang Gu for assistance in collecting and feeding the rice stem borer. The authors also sincerely thank Professor Xiao-Qiang Yu working at University of Missouri for giving some suggestions in revising and polishing the manuscript.

\section{Author details}

'State Key Laboratory of Rice Biology \& Key Laboratory of Agricultural Entomology of Ministry of Agriculture, Institute of Insect Sciences, Zhejiang University, Hangzhou 310058, China. ${ }^{2}$ State \& Local Joint Engineering Research Center of Green Pesticide Invention and Application, College of Plant Protection, Nanjing Agricultural University, Nanjing 210095, China. ${ }^{3}$ Institute of Insect Sciences, Zhejiang University, Hangzhou 310058, China.

Received: 19 January 2015 Accepted: 30 June 2015

Published online: 15 July 2015 


\section{References}

1. McCoole MD, D'Andrea BT, Baer KN, Christie AE. Genomic analyses of gas (nitric oxide and carbon monoxide) and small molecule transmitter (acetylcholine, glutamate and GABA) signaling systems in Daphnia pulex. Comp Biochem Phys D. 2012;7:124-60.

2. Chen LJ, Sun BH, Cao Y, Yao HD, Qu JP, Liu C, et al. The effects of avermectin on amino acid neurotransmitters and their receptors in the pigeon brain. Pestic Biochem Phys. 2014;110:13-9.

3. Pohanka M. Alpha7 nicotinic acetylcholine receptor is a target in pharmacology and toxicology. Int J Mol Sci. 2012;13:2219-38.

4. Jones AK, Sattelle DB. The cys-loop ligand-gated ion channel gene superfamily of the red flour beetle, Tribolium castaneum. BMC Genomics. 2007;8:327.

5. Wu SF, Xu G, Qi YX, Xia RY, Huang J, Ye GY. Two splicing variants of a novel family of octopamine receptors with different signaling properties. J Neurochem. 2014;129:37-47.

6. Blenau W, Baumann A. Molecular and pharmacological properties of insect biogenic amine receptors: Lessons from Drosophila melanogaster and Apis mellifera. Arch Insect Biochem Physiol. 2001;48:13-38.

7. Wu SF, Sun FD, Qi YX, Yao Y, Fang Q, Huang J, et al. Parasitization by Cotesia chilonis influences gene expression in fatbody and hemocytes of Chilo suppressalis. PLoS One. 2013;8:e74309-9.

8. Wu M, Zhang S, Yao R, Wu SF, Su JY, Gao CF. Susceptibility of the rice stem borer, Chilo suppressalis (Lepidoptera: Crambidae), to flubendiamide in China. J Econ Entomol. 2014;107:1250-5.

9. Su JY, Zhang ZZ, Wu M, Gao CF. Changes in insecticide resistance of the rice striped stem borer (Lepidoptera: Crambidae). J Econ Entomol. 2014; 107:333-41

10. He YP, Zhang JF, Chen JM. Effect of synergists on susceptibility to chlorantraniliprole in field populations of Chilo suppressalis (Lepidoptera: Pyralidae). J Econ Entomol. 2014;107:791-6.

11. Huang J, Wu SF, Li XH, Adamo SA, Ye GY. The characterization of a concentration-sensitive a-adrenergic-like octopamine receptor found on insect immune cells and its possible role in mediating stress hormone effects on immune function. Brain Behav Immun. 2012;26:942-50.

12. Wu SF, Yao Y, Huang J, Ye GY. Characterization of a $\beta$-adrenergic-like octopamine receptor from the rice stem borer (Chilo suppressalis). J Exp Biol. 2012;215:2646-52.

13. Wu SF, Huang J, Ye GY. Molecular cloning and pharmacological characterisation of a tyramine receptor from the rice stem borer, Chilo suppressalis (Walker). Pest Manag Sci. 2013;69:126-34.

14. Wu SF, Xu G, Ye GY. Characterization of a tyramine receptor type 2 from hemocytes of rice stem borer, Chilo suppressalis. J Insect Physiol. 2015:75:39-46.

15. Hui XM, Yang LW, He GL, Yang QP, Han ZJ, Li F. RNA interference of ace1 and ace 2 in Chilo suppressalis reveals their different contributions to motor ability and larval growth. Insect Mol Biol. 2011;20:507-18.

16. Grabherr MG, Haas BJ, Yassour M, Levin JZ, Thompson DA, Amit I, et al. Full-length transcriptome assembly from RNA-Seq data without a reference genome. Nat Biotechnol. 2011;29:644-52.

17. Gotz S, Garcia-Gomez JM, Terol J, Williams TD, Nagaraj SH, Nueda MJ, et al. High-throughput functional annotation and data mining with the Blast2GO suite. Nucleic Acids Res. 2008;36:3420-35.

18. Finn RD, Bateman A, Clements J, Coggill P, Eberhardt RY, Eddy SR, et al. Pfam: the protein families database. Nucleic Acids Res. 2014:42:D222-30.

19. Altschul SF, Madden TL, Schaffer AA, Zhang J, Zhang Z, Miller W, et al. Gapped BLAST and PSI-BLAST: a new generation of protein database search programs. Nucleic Acids Res. 1997;25:3389-402.

20. Liu TM, Zhu SY, Tang QM, Chen P, Yu YT, Tang SW. De novo assembly and characterization of transcriptome using Illumina paired-end sequencing and identification of CesA gene in ramie (Boehmeria nivea L. Gaud). BMC Genomics. 2013;14:125.

21. Mao XZ, Cai T, Olyarchuk JG, Wei LP. Automated genome annotation and pathway identification using the KEGG Orthology (KO) as a controlled vocabulary. Bioinformatics. 2005;21:3787-93.

22. Ye XD, Su YL, Zhao QY, Xia WQ, Liu SS, Wang XW. Transcriptomic analyses reveal the adaptive features and biological differences of guts from two invasive whitefly species. BMC Genomics. 2014;15:370.

23. Wu SF, Guo JY, Huang J, Ye GY. Advances in insect octopamine and tyramine. Acta Entomol Sin. 2010;53:1157-66.
24. Donly BC, Caveney S. A transporter for phenolamine uptake in the arthropod CNS. Arch Insect Biochem Physiol. 2005;59:172-83.

25. Draper I, Kurshan PT, McBride E, Jackson FR, Kopin AS. Locomotor activity is regulated by D2-like receptors in Drosophila: an anatomic and functional analysis. Dev Neurobiol. 2007;67:378-93.

26. Zhang K, Guo JZ, Peng Y, Xi W, Guo AK. Dopamine-mushroom body circuit regulates saliency-based decision-making in Drosophila. Science. 2007;316:1901-4

27. Ma ZY, Guo W, Guo XJ, Wang XH, Kang L. Modulation of behavioral phase changes of the migratory locust by the catecholamine metabolic pathway. Proc Natl Acad Sci U S A. 2011;108:3882-7.

28. Crickmore MA, Vosshall LB. Opposing dopaminergic and GABAergic neurons control the duration and persistence of copulation in Drosophila. Cell. 2013;155:881-93.

29. Marella S, Mann K, Scott K. Dopaminergic modulation of sucrose acceptance behavior in Drosophila. Neuron. 2012;73:941-50.

30. Agarwal M, Giannoni Guzman M, Morales-Matos C, Del Valle Diaz RA, Abramson Cl, Giray T. Dopamine and octopamine influence avoidance learning of honey bees in a place preference assay. PLoS One. 2011;6: e25371.

31. Christie AE, Fontanilla TM, Roncalli V, Cieslak MC, Lenz PH. Identification and developmental expression of the enzymes responsible for dopamine, histamine, octopamine and serotonin biosynthesis in the copepod crustacean Calanus finmarchicus. Gen Comp Endocrinol. 2014;195:28-39.

32. True JR, Yeh SD, Hovemann BT, Kemme T, Meinertzhagen IA, Edwards TN, et al. Drosophila tan encodes a novel hydrolase required in pigmentation and vision. PLoS Genet. 2005;1: e63.

33. Greer CL, Grygoruk A, Patton DE, Ley B, Romero-Calderon R, Chang HY, et al. A splice variant of the Drosophila vesicular monoamine transporter contains a conserved trafficking domain and functions in the storage of dopamine, serotonin, and octopamine. J Neurobiol. 2005:64:239-58.

34. Ritz MC, Cone EJ, Kuhar MJ. Cocaine inhibition of ligand binding at dopamine, norepinephrine and serotonin transporters: a structure-activity study. Life Sci. 1990;46:635-45.

35. Chang HY, Grygoruk A, Brooks ES, Ackerson LC, Maidment NT, Bainton RJ, et al. Overexpression of the Drosophila vesicular monoamine transporter increases motor activity and courtship but decreases the behavioral response to cocaine. Mol Psychiatry. 2006;11:99-113.

36. Vie A, Cigna M, Toci R, Birman S. Differential regulation of Drosophila tyrosine hydroxylase isoforms by dopamine binding and CAMP-dependent phosphorylation. J Biol Chem. 1999;274:16788-95.

37. Sarkar C, Basu B, Chakroborty D, Dasgupta PS, Basu S. The immunoregulatory role of dopamine: an update. Brain Behav Immun. 2010:24:525-8.

38. Huang J, Hamasaki T, Ozoe F, Ohta H, Enomoto Kl, Kataoka H, et al. Identification of critical structural determinants responsible for octopamine binding to the a-adrenergic-like Bombyx mori octopamine receptor. Biochemistry. 2007:46:5896-903.

39. Huang J, Hamasaki T, Ozoe Y. Pharmacological characterization of a Bombyx mori a-adrenergic-like octopamine receptor stably expressed in a mammalian cell line. Arch Insect Biochem Physiol. 2010;73:74-86.

40. Huang J, Ohta H, Inoue N, Takao H, Kita T, Ozoe F, et al. Molecular cloning and pharmacological characterization of a Bombyx mori tyramine receptor selectively coupled to intracellular calcium mobilization. Insect Biochem Mol Biol. 2009;39:842-9.

41. Roeder T. Tyramine and octopamine: ruling behavior and metabolism. Annu Rev Entomol. 2005;50:447-77.

42. Huang J, Hamasaki T, Ozoe F, Ozoe Y. Single amino acid of an octopamine receptor as a molecular switch for distinct $\mathrm{G}$ protein couplings. Biochem Biophys Res Commun. 2008;371:610-4

43. Roeder T. Octopamine in invertebrates. Prog Neurobiol. 1999:59:533-61.

44. Adamo SA. Why should an immune response activate the stress response? Insights from the insects (the cricket Gryllus texensis). Brain Behav Immun. 2010;24:194-200

45. Wang Q, He MX. Molecular characterization and analysis of a putative $5-\mathrm{HT}$ receptor involved in reproduction process of the pearl oyster Pinctada fucata. Gen Comp Endocrinol. 2014;204:71-9.

46. Vleugels R, Lenaerts C, Baumann A, Vanden Broeck J, Verlinden H. Pharmacological characterization of a $5-\mathrm{HT}_{1}$-type serotonin receptor in the red flour beetle, Tribolium castaneum. PLoS One. 2013;8: e65052. 
47. Qi YX, Xia RY, Wu YS, Stanley D, Huang J, Ye GY. Larvae of the small white butterfly, Pieris rapae, express a novel serotonin receptor. J Neurochem. 2014; 131:767-77

48. Kristensen AS, Andersen J, Jorgensen TN, Sorensen L, Eriksen J, Loland CJ, et al. SLC6 neurotransmitter transporters: structure, function, and regulation. Pharmacol Rev. 2011:63:585-640.

49. Coleman CM, Neckameyer WS. Serotonin synthesis by two distinct enzymes in Drosophila melanogaster. Arch Insect Biochem Physiol. 2005;59:12-31.

50. Watanabe T, Sadamoto H, Aonuma H. Identification and expression analysis of the genes involved in serotonin biosynthesis and transduction in the field cricket Gryllus bimaculatus. Insect Mol Biol. 2011;20:619-35.

51. Ahern GP. 5-HT and the immune system. Curr Opin Pharmacol. 2011;11:29-33.

52. Kanof PD, Greengard P. Brain histamine receptors as targets for antidepressant drugs. Nature. 1978;272:329-33.

53. Witte I, Kreienkamp HJ, Gewecke M, Roeder T. Putative histamine-gated chloride channel subunits of the insect visual system and thoracic ganglion. J Neurochem. 2002;83:504-14.

54. Stuart AE. From fruit flies to barnacles, histamine is the neurotransmitter of arthropod photoreceptors. Neuron. 1999;22:431-3.

55. Gisselmann G, Pusch H, Hovemann BT, Hatt H. Two cDNAs coding for histamine-gated ion channels in D. melanogaster. Nat Neurosci. 2002;5:11-2.

56. Hong ST, Bang S, Paik D, Kang J, Hwang S, Jeon K, et al. Histamine and its receptors modulate temperature-preference behaviors in Drosophila. J Neurosci. 2006;26:7245-56.

57. Oh Y, Jang D, Sonn JY, Choe J. Histamine-HisCl1 receptor axis regulates wake-promoting signals in Drosophila melanogaster. PLoS One. 2013;8: e68269.

58. Nassel DR. Histamine in the brain of insects: a review. Microsc Res Tech. 1999:44:121-36

59. Perdan-Pirkmajer K, Pirkmajer S, Cerne K, Krzan M. Molecular and kinetic characterization of histamine transport into adult rat cultured astrocytes. Neurochem Int. 2012;61:415-22.

60. Ogasawara M, Yamauchi K, Satoh Y, Yamaji R, Inui K, Jonker JW, et al. Recent advances in molecular pharmacology of the histamine systems: organic cation transporters as a histamine transporter and histamine metabolism. J Pharmacol Sci. 2006;101:24-30.

61. Dupuis J, Louis T, Gauthier M, Raymond V. Insights from honeybee (Apis mellifera) and fly (Drosophila melanogaster) nicotinic acetylcholine receptors: from genes to behavioral functions. Neurosci Biobehav Rev. 2012;36:1553-64

62. Shao YM, Dong K, Zhang CX. The nicotinic acetylcholine receptor gene family of the silkworm, Bombyx mori. BMC Genomics. 2007;8:324.

63. Collin C, Hauser F, de Valdivia EG, Li S, Reisenberger J, Carlsen EMM, et al. Two types of muscarinic acetylcholine receptors in Drosophila and other arthropods. Cell Mol Life Sci. 2013;70:3231-42.

64. Rotundo RL. Expression and localization of acetylcholinesterase at the neuromuscular junction. J Neurocytol. 2003;32:743-66.

65. Gabrielle P, Jeana M, Lorenza EC. Cytosolic choline acetyltransferase binds specifically to cholinergic plasma membrane of rat brain synaptosomes to generate membrane-bound enzyme. Neurochem Res. 2003;28:543-9.

66. McLean H, Verellen L, Caveney S, Donly C. Molecular cloning and functional characterization of a neuronal choline transporter from Trichoplusia ni. Insect Biochem Mol Biol. 2005;35:61-72.

67. Kim YH, Lee $\mathrm{SH}$. Which acetylcholinesterase functions as the main catalytic enzyme in the Class Insecta? Insect Biochem Mol Biol. 2013;43:47-53.

68. Zhang $Y X$, Shao $Y$, Jiang F, Li J, Liu ZW. Identification of two acetylcholinesterases in Pardosa pseudoannulata and the sensitivity to insecticides. Insect Biochem Mol Biol. 2014;46:25-30.

69. Lu YH, Park Y, Gao XW, Zhang X, Yao JX, Pang YP, et al. Cholinergic and non-cholinergic functions of two acetylcholinesterase genes revealed by gene-silencing in Tribolium castaneum. Sci Rep. 2012;2:288.

70. Lu YH, Pang YP, Park Y, Gao XW, Yao JX, Zhang X, et al. Genome organization, phylogenies, expression patterns, and three-dimensional protein models of two acetylcholinesterase genes from the red flour beetle. PLoS One. 2012;7: e32288.

71. Wessler I, Kirkpatrick CJ. Acetylcholine beyond neurons: the non-neuronal cholinergic system in humans. Br J Pharmacol. 2008;154:1558-71.

72. Kita T, Ozoe F, Ozoe Y. Expression pattern and function of alternative splice variants of glutamate-gated chloride channel in the housefly Musca domestica. Insect Biochem Mol Biol. 2014;45:1-10.
73. Panek I, Torkkeli PH. Inhibitory glutamate receptors in spider peripheral mechanosensory neurons. Eur J Neurosci. 2005;22:636-46.

74. Liu HP, Lin SC, Lin CY, Yeh SR, Chiang AS. Glutamate-gated chloride channels inhibit juvenile hormone biosynthesis in the cockroach, Diploptera punctata. Insect Biochem Mol Biol. 2005;35:1260-8.

75. McCarthy EV, Wu Y, Decarvalho T, Brandt C, Cao G, Nitabach MN. Synchronized bilateral synaptic inputs to Drosophila melanogaster neuropeptidergic rest/arousal neurons. J Neurosci. 2011;31:8181-93.

76. El Hassani AK, Schuster S, Dyck Y, Demares F, Leboulle G, Armengaud C. Identification, localization and function of glutamategated chloride channel receptors in the honeybee brain. Eur J Neurosci. 2012;36:2409-20.

77. Wolstenholme AJ. Glutamate-gated Chloride Channels. J Biol Chem. 2012;287:40232-8.

78. Besson MT, Sinakevitch I, Melon C, Iche-Torres M, Birman S. Involvement of the Drosophila taurine/aspartate transporter dEAAT2 in selective olfactory and gustatory perceptions. J Comp Neurol. 2011;519:2734-57.

79. Kita T, Ozoe F, Azuma M, Ozoe Y. Differential distribution of glutamate- and GABA-gated chloride channels in the housefly Musca domestica. J Insect Physiol. 2013;59:887-93.

80. Pool AH, Kvello P, Mann K, Cheung SK, Gordon MD, Wang L, et al. Four GABAergic interneurons impose feeding restraint in Drosophila. Neuron. 2014;83:164-77.

81. Dimitrijevic N, Dzitoyeva S, Satta R, Imbesi M, Yildiz S, Manev H. Drosophila $G_{B B A}$ receptors are involved in behavioral effects of $\gamma$-hydroxybutyric acid (GHB). Eur J Pharmacol. 2005;519:246-52.

82. Agosto J, Choi JC, Parisky KM, Stilwell G, Rosbash M, Griffith LC. Modulation of $\mathrm{GABA}_{\mathrm{A}}$ receptor desensitization uncouples sleep onset and maintenance in Drosophila. Nat Neurosci. 2008:11:354-9.

83. Hamasaka Y, Wegener C, Nassel DR. GABA modulates Drosophila circadian clock neurons via GABA receptors and decreases in calcium. J Neurobiol. 2005;65:225-40.

84. Dzitoyeva S, Dimitrijevic N, Manev H. Y-aminobutyric acid B receptor 1 mediates behavior-impairing actions of alcohol in Drosophila: adult RNA interference and pharmacological evidence. Proc Natl Acad Sci U S A. 2003;100:5485-90

85. Boumghar K, Couret-Fauvel T, Garcia M, Armengaud C. Evidence for a role of GABA- and glutamate-gated chloride channels in olfactory memory. Pharmacol Biochem Behav. 2012;103:69-75.

86. Ozoe Y. Y-Aminobutyrate- and glutamate-gated chloride channels as targets of insecticides. Adv Insect Physiol. 2013:44:211-86.

87. Ilg T, Berger M, Noack S, Rohwer A, Gassel M. Glutamate decarboxylase of the parasitic arthropods Ctenocephalides felis and Rhipicephalus microplus: gene identification, cloning, expression, assay development, identification of inhibitors by high throughput screening and comparison with the orthologs from Drosophila melanogaster and mouse. Insect Biochem Mol Biol. 2013:43:162-77.

88. Fenalti G, Law RH, Buckle AM, Langendorf C, Tuck K, Rosado CJ, et al. GABA production by glutamic acid decarboxylase is regulated by a dynamic catalytic loop. Nat Struct Mol Biol. 2007;14:280-6.

89. Boudko DY. Molecular basis of essential amino acid transport from studies of insect nutrient amino acid transporters of the SLC6 family (NAT-SLC6). J Insect Physiol. 2012;58:433-49.

90. Han LZ, Li SB, Liu PL, Peng YF, Hou ML. New artificial diet for continuous rearing of Chilo suppressalis (Lepidoptera: Crambidae). Ann Entomol Soc Am. 2012;105:253-8.

91. Fumagalli D, Blanchet-Cohen A, Brown D, Desmedt C, Gacquer D, Michiels $S$, et al. Transfer of clinically relevant gene expression signatures in breast cancer: from Affymetrix microarray to Illumina RNA-Sequencing technology. BMC Genomics. 2014;15:1008.

92. Gu SH, Wu KM, Guo YY, Pickett JA, Field LM, Zhou JJ, et al. Identification of genes expressed in the sex pheromone gland of the black cutworm Agrotis ipsilon with putative roles in sex pheromone biosynthesis and transport. BMC Genomics. 2013;14:636.

93. Tamura K, Peterson D, Peterson N, Stecher G, Nei M, Kumar S. MEGA5 molecular evolutionary genetics analysis using maximum likelihood, evolutionary distance, and maximum parsimony methods. Mol Biol Evol. 2011:28:2731-9.

94. Larkin MA, Blackshields G, Brown NP, Chenna R, McGettigan PA, McWilliam $H$, et al. Clustal $W$ and Clustal $X$ version 2.0. Bioinformatics. 2007;23:2947-8. 
95. Livak KJ, Schmittgen TD. Analysis of relative gene expression data using real-time quantitative PCR and the $2^{-\Delta \Delta C T}$ method. Methods. 2001;25:402-8.

96. Tang QY, Zhang CX. Data Processing System (DPS) software with experimental design, statistical analysis and data mining developed for use in entomological research. Insect Sci. 2013;20:254-60.

Submit your next manuscript to BioMed Central and take full advantage of:

- Convenient online submission

- Thorough peer review

- No space constraints or color figure charges

- Immediate publication on acceptance

- Inclusion in PubMed, CAS, Scopus and Google Scholar

- Research which is freely available for redistribution 